Groups Geom. Dyn. 4 (2010), 127-162

DOI $10.4171 / \mathrm{GGD} / 77$
Groups, Geometry, and Dynamics

(C) European Mathematical Society

\title{
Equivariant bundles and isotropy representations
}

\author{
Ian Hambleton and Jean-Claude Hausmann*
}

\begin{abstract}
We introduce a new construction, the isotropy groupoid, to organize the orbit data for split $\Gamma$-spaces. We show that equivariant principal $G$-bundles over split $\Gamma$-CW complexes $X$ can be effectively classified by means of representations of their isotropy groupoids. For instance, if the quotient complex $A=\Gamma \backslash X$ is a graph, with all edge stabilizers toral subgroups of $\Gamma$, we obtain a purely combinatorial classification of bundles with structural group $G$ a compact connected Lie group. If $G$ is abelian, our approach gives combinatorial and geometric descriptions of some results of Lashof-May-Segal [18] and Goresky-Kottwitz-MacPherson [10].
\end{abstract}

Mathematics Subject Classification (2010). 55R91, 55R15; 22A22.

Keywords. Equivariant bundles.

\section{Introduction}

In this paper we continue our study of equivariant principal bundles via isotropy representations (see [13]). If $\Gamma$ and $G$ are topological groups, then a $\Gamma$-equivariant principal $G$-bundle is a locally trivial, principal $G$-bundle $p: E \rightarrow X$ such that $E$ and $X$ are left $\Gamma$-spaces. The projection map $p$ is $\Gamma$-equivariant and $\gamma(e \cdot g)=(\gamma e) \cdot g$, where $\gamma \in \Gamma$ and $g \in G$ acts on $e \in E$ by the principal action. Equivariant principal bundles, and their natural generalizations, were studied by T. E. Stewart [25], T. tom Dieck [26], [27], I (8.7), R. Lashof [15], [16] together with P. May [17] and G. Segal [18].

The isotropy representation at a point $x \in X$ is the homomorphism $\alpha_{x}: \Gamma_{x} \rightarrow G$ defined by the formula

$$
\gamma \cdot \tilde{x}=\tilde{x} \cdot \alpha_{x}(\gamma)
$$

where $\tilde{x} \in p^{-1}(x)$. The homomorphism $\alpha_{x}$ is independent of the choice of $\tilde{x}$ up to conjugation in $G$. Here $\Gamma_{x}$ denotes the isotropy subgroup or stabilizer of $x \in X$.

The use of isotropy representations is particularly effective when the projection $\pi: X \rightarrow \Gamma \backslash X \stackrel{\approx}{\longrightarrow} A$ has a section $\varphi: A \rightarrow X$. We call the triple $(X, \pi, \varphi)$ a

\footnotetext{
* Research partially supported by NSERC Discovery Grant A4000. The authors would like to thank the Max Planck Institut für Mathematik for its hospitality and support while working on this paper.
} 
split $\Gamma$-space over $A$. A natural source of examples is symplectic toric manifolds (see (4.8)), where $A$ is the moment polytope. Under reasonable assumptions, a split $\Gamma$-space over $A$ is uniquely determined by its isotropy groupoid

$$
\mathcal{I}:=\left\{(\gamma, a) \in \Gamma \times A \mid \gamma \in \Gamma_{\varphi(a)}\right\}
$$

(see Proposition 2.1). A $\Gamma$-equivariant principal $G$-bundle $\eta:=(E \stackrel{p}{\rightarrow} X)$ is called split if the pull-back $\varphi^{*}(\eta)$ is a trivial bundle. The isotropy representations of $\eta$ then produce a continuous groupoid representation of $\mathcal{I}$ in $G$ which is well defined up to conjugation by $\operatorname{Map}(A, G)$. We denote by

$$
\operatorname{Rep}^{G}(\mathcal{I})=\operatorname{Hom}(\mathcal{I}, G) / \operatorname{Map}(A, G)
$$

the space of conjugacy classes of such groupoid representations. The first part of this paper is devoted to proving the following general classification theorem.

Theorem A. Suppose that $\Gamma$ and $G$ are compact Lie groups. Let $X$ be split $\Gamma$-space over A with isotropy groupoid $\mathcal{I}$. Assume that $A$ is locally compact, and that $\mathcal{I}$ is locally maximal. Then the equivalence classes of split $\Gamma$-equivariant $G$-bundles over $X$ are in bijection with $\operatorname{Rep}^{G}(\mathcal{I})$.

The relevant definitions are given in Section 3: see $\S 3 \mathrm{~A}$ for equivariant bundles, $\S 3 \mathrm{~B}$ for the notion of a locally maximal isotropy groupoid, and the proof of Theorem A is given in $\S 3 \mathrm{C}$. In our applications we will assume that $X$ is a $\Gamma$-CW-complex, equipped with a splitting $\varphi: A \rightarrow X$ such that $\Gamma_{\varphi(a)}$ is constant on each open cell of its quotient $\mathrm{CW}$-complex $A$. This property doesn't always hold (see Remark 4.2), but it seems a natural assumption. We call the resulting isotropy groupoids cellular (see $\S 4 \mathrm{~A}$ ). A cellular groupoid is a combinatorial object and, when $\Gamma$ is discrete, it is a particular case of a developable simple complex of groups as considered by M. Bridson and A. Haefliger [4]. Cellular groupoids whose stalks $\mathcal{I}_{a}$ are compact Lie groups are called proper groupoids. They arise from proper actions of a Lie group $\Gamma$, as studied for example in [20] for $\Gamma$ discrete. In Theorem 4.5 we extend Theorem A to the classification of split $\Gamma$-equivariant bundles over a proper groupoid.

In a second part of this paper, we describe some approaches to computing $\operatorname{Rep}^{G}(\mathcal{I})$ assuming $\mathcal{I}$ is cellular. There is a corresponding notion of cellular representations, meaning those which are constant on the open cells of $A$, whose classes modulo conjugation by a fixed element of $G$ form a set denoted by $\operatorname{Rep}_{\text {cell }}^{G}(\mathcal{I})$ (see $\S 5 \mathrm{~A}$ ). The cellular representations are also purely combinatorial, and for $A$ a regular CW-complex, $\operatorname{Rep}_{\text {cell }}^{G}(\mathcal{I})$ is determined by restriction to the 0-skeleton of $A$ (see Proposition 5.1). We consider Theorem A to be an effective method of classifying equivariant bundles whenever $\operatorname{Rep}^{G}(\mathcal{I})$ can be reduced to $\operatorname{Rep}_{\text {cell }}^{G}(\mathcal{I})$. We therefore study the natural map $\operatorname{Rep}_{\text {cell }}^{G}(\mathcal{I}) \rightarrow \operatorname{Rep}^{G}(\mathcal{I})$, which turns out to be injective (Proposition 5.18) but not surjective in general (see (5.21)). It is however bijective when $G$ is compact abelian (Proposition 5.3), or when $A$ is a tree (Proposition 5.20). 
We next consider the case where $A$ is a graph. Here it is useful to define a related object $\overline{\operatorname{Rep}}_{\text {cell }}^{G}(\mathcal{I})$, by allowing conjugation of cellular homomorphisms over each cell of $A$ independently. It turns out that there exists a natural map $l: \operatorname{Rep}^{G}(\mathcal{I}) \rightarrow$ $\overline{\operatorname{Rep}}_{\text {cell }}^{G}(\mathcal{I})$. In Theorem 5.11, we study this map for $G$ a compact connected Lie group. A sample application of Theorem 5.11 is given by the following:

Theorem B. Let $\Gamma$ and $G$ be a compact Lie groups, with $G$ connected, and suppose that $A$ is a graph. If $\mathcal{I}$ is a cellular groupoid with all edge stabilizers torus subgroups of $\Gamma$, then the map $\imath: \operatorname{Rep}^{G}(\mathcal{I}) \rightarrow \overline{\operatorname{Rep}}_{\text {cell }}^{G}(\mathcal{I})$ is a bijection.

Recall that there exists a classifying space $B(\Gamma, G)$ for $\Gamma$-equivariant principal $G$-bundle [26], so the classification of equivariant bundles in particular cases can also be approached by studying the $\Gamma$-equivariant homotopy type of $B(\Gamma, G)$. If the structural group $G$ of the bundle is abelian, then the main result of Lashof, May and Segal [18] states that equivariant bundles over a $\Gamma$-space $X$ are classified by the ordinary homotopy classes of maps $\left[X \times_{\Gamma} E \Gamma, B G\right]$. For non-abelian structural groups, it appears that the natural map $\theta:[X, B(\Gamma, G)]_{\Gamma} \rightarrow\left[X \times_{\Gamma} E \Gamma, B G\right]$ misses a lot of information, and our results could be interpreted as studying $\theta^{-1}(\bullet)$.

Our isotropy groupoid $\mathcal{I}$ has a classifying space $B \mathcal{I}$ constructed by Haefliger [12], p. 140. We observe that $B \mathcal{I} \simeq X \times_{\Gamma} E \Gamma$ when $\mathcal{I}$ is cellular. In our language, the result of [18] implies that the natural map $B: \operatorname{Rep}^{G}(\mathcal{I}) \rightarrow[B \mathcal{I}, B G]$ is injective for $G$ compact abelian. More generally, we show in Corollary 6.4:

Theorem C. Let $G$ and $\Gamma$ be compact Lie groups, with $G$ abelian. Let $X$ be a split $\Gamma$-space over $A$ with cellular isotropy groupoid $\mathcal{I}$. Suppose that $H^{1}\left(A ; \pi_{0}(G)\right)=$ $H^{2}(A ; \mathbb{Z})=0$. Then the map $B: \operatorname{Rep}^{G}(\mathcal{I}) \rightarrow[B \mathcal{I}, B G]$ is a bijection.

In 6.5 we point out the connection between our classification results and equivariant K-theory. Finally, in 6.7, we compare our results with some classical theorems in equivariant cohomology, due to Chang-Skjelbred [5] and Goresky-KottwitzMacPherson [10].

\section{Preliminaries}

Most of this section contains folklore facts about compact Lie groups. Let $K$ and $G$ be topological groups. We denote by $\operatorname{Hom}(K, G)$ the space of continuous homomorphisms from $K$ to $G$, endowed with the compact-open topology. Two homomorphisms $\alpha_{1}, \alpha_{2} \in \operatorname{Hom}(K, G)$ are called conjugate if there exists $g \in G$ such that $\alpha_{2}(\gamma)=g^{-1} \alpha_{1}(\gamma) g$ for all $\gamma \in K$. We denote by $\overline{\operatorname{Hom}}(K, G)$ the space of conjugacy classes, endowed with the quotient topology.

Lemma 1.1. Let $K$ and $G$ be compact Lie groups. Then the space $\overline{\operatorname{Hom}}(K, G)$ is totally disconnected. 
Proof. A bi-invariant Riemannian metric on $G$ gives rise to a bi-invariant distance $d$ on $G$. The uniform convergence distance on $\operatorname{Hom}(K, G)$,

$$
d(\alpha, \beta)=\max _{k \in K} d(\alpha(k), \beta(k)),
$$

induces the compact-open topology. Let us denote by $\bar{\alpha}, \bar{\beta} \in \overline{\operatorname{Hom}}(K, G)$ the conjugacy classes of $\alpha$ and $\beta$. One checks that the formula

$$
\bar{d}(\bar{\alpha}, \bar{\beta})=\min _{g \in G} d\left(g \alpha g^{-1}, \beta\right)=\min _{g, h \in G} d\left(g \alpha g^{-1}, h \beta h^{-1}\right)
$$

defines a distance on $\overline{\operatorname{Hom}}(K, G)$. Because $\bar{d}(\bar{\alpha}, \bar{\beta}) \leq d(\alpha, \beta)$, the projection $p:(\operatorname{Hom}(K, G), d) \rightarrow(\overline{\operatorname{Hom}}(K, G), \bar{d})$ is continuous, so the quotient topology on $\operatorname{Hom}(K, G)$ is finer than the metric topology (one can check that they are equal, but we shall not use that).

The space $\overline{\operatorname{Hom}}(K, G)$ has at most countably many points [2], Prop. 10.14. Therefore, the set $\mathscr{D}=\{d(a, b) \mid a, b \in \overline{\operatorname{Hom}}(K, G)\}$ is at most countable. Let $a, b \in \overline{\operatorname{Hom}}(K, G)$ with $a \neq b$. There exists $\lambda \in \mathbb{R}$ with $0<\lambda<\bar{d}(a, b)$ and $\lambda \notin D$. The space $\overline{\operatorname{Hom}}(K, G)$ is then the disjoint union of $\{x \mid \bar{d}(a, x)<\lambda\}$ and $\{x \mid \bar{d}(a, x)>\lambda\}$. These are non-empty open sets for the topology induced by $\bar{d}$ and then for the quotient topology. This proves that any subspace of $\overline{\operatorname{Hom}}(K, G)$ containing more than one point is not connected.

Lemma 1.2. Let $K$ and $G$ be compact Lie groups. Let $B$ be a space homeomorphic to a compact disk and let $b \in B$. Let $x \mapsto \beta_{x}$ be a continuous map from $B$ to $\operatorname{Hom}(K, G)$. Then there is a continuous $x \mapsto g_{x}$ from $B$ to $G$ with $g_{b}=1$ such that $\beta_{x}(\gamma)=g_{x}^{-1} \beta_{b}(\gamma) g_{x}$ for all $\gamma \in K$ and all $x \in B$.

Proof. If $B$ is of dimension $n$, then, by a pointed homeomorphism, one can replace the pair $(B, b)$ by $\left([0,1]^{n}, 0\right)$ if $b$ lies in the boundary of $B$, or by $\left([-1,1]^{n}, 0\right)$ otherwise. By Lemma 1.1, $\beta_{x}$ stays for all $x$ in the same conjugacy class $\mathcal{O}$ of $\operatorname{Hom}(K, G)$. As seen in the proof of Lemma 1.1, the space $\operatorname{Hom}(K, G)$ is metric, therefore Hausdorff. Therefore, $\mathcal{O}$ is compact. As $G$ is compact, the map $p: G \rightarrow \mathcal{O}$ given by $g \mapsto g \beta_{0} g^{-1}$ can then be identified with a principal bundle whose structure group is the centraliser $\mathcal{Z}\left(\beta_{0}(K)\right)$, which is a closed subgroup of $G$. Lemma 1.2 then follows from the a recursive use of the homotopy lifting property.

Lemma 1.3. Let $K$ be a compact abelian Lie group. Denote by $K_{1}$ the connected component of the unit element $1 \in K$. Then there is a bicontinuous isomorphism $K_{1} \times \pi_{0}(K) \stackrel{\approx}{\rightarrow} K$.

Proof. As $K$ is abelian, it suffices to construct a homomorphic section of the projection $K \rightarrow \pi_{0}(K)$. As $\pi_{0}(K)$ is finite, one can reduce to the case where $\pi_{0}(K)=C$ is a cyclic group of order $m$. Let $c \in C$ be a generator and choose $\tilde{c} \in K$ representing $c$. Then $\tilde{c}^{m}$ is in $K_{1}$ and there exists $\gamma \in K_{1}$ such that $\gamma^{m}=\tilde{c}^{m}$. The map $\sigma: C \rightarrow G$ defined by $\sigma\left(c^{k}\right)=\tilde{c}^{k} \gamma^{-k}$ is a homomorphic section of the projection $K \rightarrow C$. 
1.4. Spaces over $A$. Let $f: X \rightarrow A$ be a continuous map between topological spaces. This enables us to consider $X$ as a "space over $A$ ". For $a \in A$, the stalk over $a$ is $X_{a}=f^{-1}(a)$. Any subspace $Y$ of $B \times A$ is seen as a space over $A$ via the projection onto $A$ restricted to $Y$.

\section{Split $\Gamma$-spaces}

Let $A$ be a topological space and $\Gamma$ be a topological group. A $\Gamma$-space is a topological space equipped with a continuous left action of $\Gamma$. If $X$ is a $\Gamma$-space and $x \in X$, we denote by $\Gamma_{x}$ the stabiliser of $x$.

A split $\Gamma$-space over $A$ is a triple $(X, \pi, \varphi)$ where

(i) $X$ is a $\Gamma$-space.

(ii) $\pi: X \rightarrow A$ is a continuous surjective map and, for each $a \in A$, the preimage $\pi^{-1}(a)$ is a single orbit.

(iii) $\varphi: A \rightarrow X$ is a continuous section of $\pi$

The maps $\pi$ and $\varphi$ may omitted from the notation and we might speak of a split $\Gamma$-space $X$ over $A$. By (ii), the map $\pi$ descends to $\bar{\pi}: \Gamma \backslash X \rightarrow A$ which is a homeomorphism (its continuous inverse is provided by the section $\varphi$ ). The map $\pi$ may thus be identified with the projection of $X$ onto the orbit space $\Gamma \backslash X$.

A ( $\Gamma, A)$-groupoid is a subspace $\mathcal{I} \subset \Gamma \times A$ such that, for each $a \in A$, the space $\mathcal{I}_{a}=\mathcal{I} \cap(\Gamma \times\{a\})$ is of the form $\tilde{I}_{a} \times\{a\}$, where $\tilde{\mathcal{I}}_{a}$ is a closed subgroup of $\Gamma$. We consider $\mathcal{I}_{a}$ as a topological group, naturally isomorphic to the closed subgroup $\tilde{\mathcal{I}}_{a}$ of $\Gamma$. We will often identify these two groups, and write, for instance, $\mathcal{I}_{a}=\mathcal{I}_{b}$ when we mean $\tilde{\mathcal{I}}_{a}=\tilde{\mathcal{I}}_{b}$. The space $\mathcal{I}$ is regarded as a topological groupoid whose space of objects is $A$ : if $a, b \in A$, the space of morphisms from $a$ to $b$ is empty when $a \neq b$ and is equal to $\mathcal{I}_{a}$ otherwise.

Let $\mathcal{I}$ be a $(\Gamma, A)$-groupoid. A (left) action of $\mathcal{I}$ on a topological space $W$ is a continuous map $\beta: \mathcal{I} \times W \rightarrow W$ such that, for each $a \in A$, the restriction of $\beta$ to $\mathcal{I}_{a} \times W$ is an action of $\mathcal{I}_{a}$ on $W$. The notation $\zeta \cdot w$ is used for $\beta(\zeta, w)$. A right action is defined accordingly, as a continuous map from $W \times \mathcal{I}$ to $W$. When $\mathcal{I}$ acts on the right on a space $V$ and on the left on a space $W$, we define the quotient space

$$
V \times \mathfrak{I} W=(V \times W) / \sim,
$$

where " $\sim$ " is the smallest equivalence relation such that $(v \cdot \zeta, w) \sim(v, \zeta \cdot w)$ for all $\zeta \in \mathcal{I}$. If $W$ is a space over $A$, then $V \times \mathcal{I} W$ is a space over $A$ as well. The stalk over $a$ is then $V \times \mathcal{I}_{a} W_{a}$.

Let $(X, \pi, \varphi)$ be a split $\Gamma$-space over $A$. Its isotropy groupoid is the $(\Gamma, A)$ groupoid defined by

$$
\mathcal{I}(X)=\mathcal{I}(X, \pi, \varphi):=\left\{(\gamma, a) \in \Gamma \times A \mid \gamma \in \Gamma_{\varphi(a)}\right\} .
$$


A ( $\Gamma, A)$-groupoid $\mathcal{I}$ is called weakly locally maximal if each point $a \in A$ admits a neighbourhood $U$ such that $\mathcal{I}_{u}$ is a subgroup of $\mathcal{I}_{a}$ for all $u \in U$. A space $X$ is called locally compact if it is Hausdorff and if every point of $X$ admits a compact neighbourhood. The main result of this section is the following proposition.

Proposition 2.1 (Reconstruction). Let $\Gamma$ be a compact topological group and $A$ be a locally compact space. Let $\mathcal{I}$ be a weakly locally maximal $(\Gamma, A)$-groupoid. Then the following properties hold.

(i) There is a split $\Gamma$-space $\left(Y_{\mathcal{I}}, \Pi, \phi\right)$ over $A$ with isotropy groupoid $\mathcal{I}$; the space $Y_{\mathcal{I}}$ is locally compact.

(ii) Let $(X, \pi, \varphi)$ and $\left(X^{\prime}, \pi^{\prime}, \varphi^{\prime}\right)$ be two split $\Gamma$-spaces over $A$ with isotropy groupoid I. Suppose that $X$ and $X^{\prime}$ are locally compact. Then there is a unique $\Gamma$-equivariant homeomorphism $F: X \rightarrow X^{\prime}$ such that $\varphi^{\prime}=F \circ \varphi$.

Proposition 2.1 permits us to speak about the split $\Gamma$-space over $A$ with isotropy groupoid $\mathcal{I}$ (as we speak about the real number field instead of $a$ real number field). The triple $\left(Y_{\mathcal{I}}, \Pi, \phi\right)$ constructed for the proof of (i) is an explicit model for this space, but other models also occur naturally, as will be seen in examples.

Proof of Proposition 2.1. The groupoid $\mathcal{I}$ acts by multiplication on the right on $\Gamma$. We let it act trivially on the left on $A$ and form the space

$$
Y_{\mathcal{I}}=\Gamma \times_{\mathcal{I}} A .
$$

The projection $\Gamma \times A \rightarrow A$ descends to a continuous surjective map $\Pi: Y_{I} \rightarrow A$. The section $\phi: A \rightarrow Y_{\mathcal{I}}$ is defined by $\phi(a)=[1, a]$, where 1 is the unit element in $\Gamma$. The $\Gamma$-action $\beta \cdot(\gamma, x)=(\beta \gamma, x)$ on $\Gamma \times A$ descends to a $\Gamma$-action on $Y_{\mathcal{I}}$. The stalk $\Pi^{-1}(a)$ is the orbit through $\phi(a)$ and $\Gamma_{\phi(a)}=\mathcal{I}_{a}$. Thus, $\left(Y_{\mathfrak{I}}, \Pi, \phi\right)$ is a split $\Gamma$-space over $A$ with isotropy groupoid $\mathcal{I}$.

To prove that $Y_{\mathfrak{I}}$ is Hausdorff, let $x$ and $y$ be two distinct points in $Y_{\mathfrak{I}}$. Let us show that they admit disjoint neighbourhoods. If $\pi(x) \neq \pi(y)$, this is obvious since $A$ is Hausdorff. In the case $\pi(x)=\pi(y)=a$, let $U$ be a neighbourhood of $a$ for which $\mathcal{I}_{b}$ is a subgroup of $\mathcal{I}_{a}$ for all $b \in U$. Then $\Pi^{-1}(U)$ is a neighbourhood of $\{x, y\}$ and there is a continuous map $f: \Pi^{-1}(U) \rightarrow\left(\Gamma_{\phi(a)} \backslash \Gamma\right) \times U$ such that $f(x) \neq f(y)$. As $\Gamma_{\phi(a)}=\mathcal{I}_{a}$ is a closed subgroup of $\Gamma$, the space $\left(\Gamma_{\phi(a)} \backslash \Gamma\right) \times U$ is Hausdorff and then $x$ and $y$ admit disjoint neighbourhoods. Observe that the proof that $Y_{\mathcal{I}}$ is Hausdorff uses only that $\Gamma$ and $A$ are Hausdorff and that $\mathcal{I}$ is weakly locally maximal.

Let $x \in Y_{\mathcal{I}}$. As $A$ is locally compact, $\Pi(x)$ admits a compact neighbourhood $V$ in $A$. Then $\Pi^{-1}(V)$ is a neighbourhood of $x$ and is the continuous image of $\Gamma \times V$ under the natural projection $\Gamma \times A \rightarrow Y_{\mathcal{I}}$. As $\Gamma \times V$ is compact and $Y_{\mathfrak{I}}$ is Hausdorff, $\Pi^{-1}(V)$ is compact. This shows that $Y_{\mathcal{I}}$ is locally compact and finishes the proof of (i).

Let us prove (ii), starting with uniqueness. Let $F_{1}, F_{2}: X \rightarrow X^{\prime}$ be two $\Gamma$ equivariant isomorphisms satisfying $F_{1} \circ \varphi=F_{2} \circ \varphi$. Then $F_{1}=F_{2}$ by equivariance, 
since $\varphi(A)$ is a fundamental domain for the $\Gamma$-action. Now, let $(X, \pi, \varphi)$ be a split $\Gamma$-space with isotropy groupoid $\mathcal{I}$. The map $\widetilde{F}: \Gamma \times A \rightarrow X$ defined by $\widetilde{F}(\gamma, a)=$ $\gamma \cdot \varphi(a)$ is continuous, $\Gamma$-equivariant and surjective. It descends to a $\Gamma$-equivariant continuous bijection $F: Y_{\mathcal{I}} \rightarrow X$. Observe that $\widetilde{F}$ is proper, that is $\widetilde{F}^{-1}(K)$ is compact for all compact subsets $K$ in $X$. Indeed, $\widetilde{F}^{-1}(K)$ is a closed subset of $\Gamma \times \pi(K)$ which is compact. Since $Y_{\mathcal{I}}$ is Hausdorff, the map $F$ is proper as well. A proper continuous bijection between locally compact spaces is a homeomorphism, which proves (b).

Remark 2.2. If in Proposition 2.1 we only assume that $\Gamma$ is locally compact, the space $Y_{\mathcal{I}}$ constructed for the proof of (i) might not be locally compact. As an example, take $A=[0,1], \mathcal{I}_{a}$ trivial for $a<1$ and $\mathcal{I}_{1}=\Gamma$. Then $Y_{\mathfrak{I}}$ is the cone on $\Gamma$, which is not locally compact if $\Gamma$ is not compact. Moreover, the uniqueness also fails in this case. Let $\Gamma=\mathbb{Z}$. Then the cone $V$ on the real integers in the complex plane, with vertex $i$ say, and the induced metric, is a split $\Gamma$-space with isotropy groupoid $\mathcal{I}$. The proof of Proposition 2.1 provides a $\Gamma$-equivariant continuous bijection from the $Y_{\mathcal{I}}$ onto $V$ but it is not a homeomorphism (a set containing one point in the interior of each segment would be closed in $Y_{\mathfrak{I}}$, even if it contains a subsequence converging to the vertex $i$ ). A version of Proposition 2.1 with $\Gamma$ non-compact is given in Proposition 4.3.

A stronger local maximality condition will play a role in Section 3. A $(\Gamma, A)$ groupoid $\mathcal{I}$ is called locally maximal if, for each point $a \in A$ and each neighbourhood $B$ of $a$, there exists an open set $U$ of $A$, with $a \in U \subset B$, and a homotopy $\rho_{t}: U \rightarrow U$ $(t \in[0,1])$ satisfying $\rho_{0}(u)=u, \rho_{1}(u)=a$ and $\mathcal{I}_{u} \subset \mathcal{I}_{\rho_{t}(u)}$ for all $u \in U$ and $t \in[0,1]$. The neighbourhood $U$ is then contractible. Locally maximal implies weakly locally maximal.

Lemma 2.3. Let $\Gamma$ be a compact topological group and $A$ be a locally compact space. Let I be a locally maximal $(\Gamma, A)$-groupoid. Let $(X, \pi, \varphi)$ be a split $\Gamma$-space over $A$ with isotropy groupoid I and let $x \in X$. Then there is a $\Gamma$-equivariant open neighbourhood $\hat{U}$ of the orbit $\Gamma x$ and $a \Gamma$-equivariant $\hat{\rho}_{t}: \hat{U} \rightarrow \widehat{U}$ such that $\hat{\rho}_{0}=\mathrm{id}$ and $\hat{\rho}_{1}(\hat{U})=\Gamma \cdot x$. Moreover, $\hat{\rho}_{t}$ satisfies $\pi \circ \hat{\rho}_{t}=\rho_{t} \circ \pi$ and $\varphi \circ \rho_{t}=\hat{\rho}_{t} \circ \varphi$.

Proof. By Proposition 2.1, one may suppose that $(X, \pi, \varphi)=\left(Y_{\mathfrak{I}}, \Pi, \phi\right)$. Let $a=$ $\Pi(x)$ and let $\rho_{t}: U \rightarrow U$ be a homotopy from an open neighbourhood $U$ of $A$ to itself, such that $\rho_{0}(u)=u, \rho_{1}(u)=a$ and $\mathcal{I}_{u} \subset \mathcal{I}_{\rho_{t}(u)}$ for all $u \in U$. Let $\hat{U}=$ $\Pi^{-1}(U)$. We check that the required homotopy $\hat{\rho}_{t}$ can be defined by $\hat{\rho}_{t}([\gamma, u]):=$ $\left[\gamma, \rho_{t}(u)\right]$.

Let $\mathcal{I}$ be a $(\Gamma, A)$-groupoid and let $\mathcal{I}^{\prime}$ be a $\left(\Gamma^{\prime}, A^{\prime}\right)$-groupoid. A morphism of 
groupoids from $\mathcal{I}$ to $\mathcal{I}^{\prime}$ is a commutative diagram

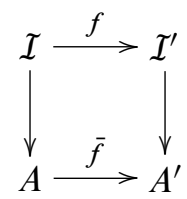

where $f$ and $\bar{f}$ are continuous maps such that, for each $a \in A$, the restriction of $f$ to $\mathcal{I}_{a}$ is a homomorphism $f_{a}: \mathcal{I}_{a} \rightarrow \mathcal{I}_{\bar{f}(a)}^{\prime}$. The map $\bar{f}$ is not mentioned when it is obvious, like an inclusion or a constant map.

\section{Split equivariant principal bundles}

§3A. Definitions. Let $G$ be a topological group and $X$ be a topological space. By a $G$-principal bundle $\eta$ over $X$, we mean, as usual, a continuous surjection $p: E \rightarrow X$ from a space $E=E(\eta)$ and a free right action $E \times G \rightarrow E$ so that $p(z \cdot g)=p(z)$, with the standard local triviality condition. Two $G$-principal bundles $\eta: E \stackrel{p}{\rightarrow} X$ and $\eta^{\prime}: E^{\prime} \stackrel{p^{\prime}}{\rightarrow} X$ over $X$ are isomorphic if there exists a $G$-homeomorphism $f: E \rightarrow E^{\prime}$ such that $p^{\prime} \circ f=p$. Isomorphism classes of $G$-principal bundles over $X$ are denoted by $\operatorname{Bun}^{G}(X)$.

Let $X$ be a $\Gamma$-space for a topological group $\Gamma$. A $G$-principal bundle $\eta: E \stackrel{p}{\rightarrow} X$ is called a $\Gamma$-equivariant principal $G$-bundle if it is given a left action $\Gamma \times E \rightarrow E$ commuting with the free right action of $G$ and such that the projection $p$ is $\Gamma$ equivariant. Two $\Gamma$-equivariant principal $G$-bundles $\eta$ and $\eta^{\prime}$ are called $\Gamma$-isomorphic (or just isomorphic) if there exists a $G$-homeomorphism from $E(\eta)$ to $E\left(\eta^{\prime}\right)$ over the identity of $X$ which is $\Gamma$-equivariant. The set of $\Gamma$-isomorphism classes of $\Gamma$ equivariant $G$-principal bundles over $X$ is denoted by $\operatorname{Bun}_{\Gamma}^{G}(X)$. There is a forgetful $\operatorname{map} \operatorname{Bun}_{\Gamma}^{G}(X) \rightarrow \operatorname{Bun}^{G}(X)$.

Let $(X, \pi, \varphi)$ is a split $\Gamma$-space over $A$ with isotropy groupoid $\mathcal{I}$. Let $\xi$ be a $\Gamma$ equivariant principal $G$-bundle over $X$. We say that $\xi$ is split if the induced bundle $\varphi^{*} \xi$ is trivial. For instance, any $\Gamma$-equivariant principal $G$-bundle is split when $A$ is contractible and paracompact, which is the case in many examples of Section $\S 4 \mathrm{~B}$. Two split $\Gamma$-equivariant principal $G$-bundles over $(X, \pi, \varphi)$ are isomorphic if they are isomorphic just as $\Gamma$-equivariant principal $G$-bundles over $X$. The set of isomorphism classes of $\Gamma$-equivariant split $G$-principal bundles over $(X, \pi, \varphi)$ is denoted by $\operatorname{SBun}_{\Gamma}^{G}(X, \pi, \varphi)$ or simply by $\operatorname{SBun}_{\Gamma}^{G}(X)$. It is a subset of $\operatorname{Bun}_{\Gamma}^{G}(X)$.

§3B. The isotropy representation. Let $\mathcal{I}$ be a $(\Gamma, A)$-groupoid and $G$ be a topological group. A continuous representation of $\mathcal{I}$ to $G$ is a continuous map $\alpha: \mathcal{I} \rightarrow G$ such that, for all $a \in A$, the restriction $\alpha_{a}$ of $\alpha$ to $\mathcal{I}_{a}$ is a homomorphism (it is thus 
a morphism of groupoids between $\mathcal{I}$ and the $(p t, G)$-groupoid $G \rightarrow p t)$. Two continuous representations $\alpha_{1}$ and $\alpha_{2}$ are called conjugate if there exists a continuous map $\psi: A \rightarrow G$ such that $\alpha_{2}(\zeta)=\psi\left(\pi_{2}(\zeta)\right)^{-1} \alpha_{1}(\zeta) \psi\left(\pi_{2}(\zeta)\right)$ for all $\zeta \in \mathcal{I}$, where $\pi_{2}: \mathcal{I} \rightarrow A$ is the second factor projection.

A continuous representation of $\alpha: \mathcal{I} \rightarrow G$ is called locally maximal if each point $a \in A$ admits a neighbourhood $U$ such that $\mathcal{I}_{u}$ is a subgroup of $\mathcal{I}_{a}$ for all $u \in U$, together with a continuous map $g: U \rightarrow G$ such that $\alpha_{u}(\gamma)=g(u) \alpha_{a}(\gamma) g(u)^{-1}$ for all $u \in U$ and all $\gamma \in \mathcal{I}_{u}$. This implies that $\mathcal{I}$ is weakly locally maximal. It is easy to see that, if $\alpha, \beta: \mathcal{I} \rightarrow G$ are two conjugate representations of $\mathcal{I}$, then $\beta$ is locally maximal if and only if $\alpha$ is weakly locally maximal. We denote by $\operatorname{Rep}^{G}(\mathcal{I})$ the set of conjugacy classes of locally maximal continuous representations of $\mathcal{I}$.

Let $(X, \pi, \varphi)$ be a split $\Gamma$-space over $A$ with isotropy groupoid $\mathcal{I}$. Let $\eta: E \stackrel{p}{\rightarrow} X$ be a split $\Gamma$-equivariant $G$-principal bundle over $X$. As $\varphi^{*} \eta$ is trivial, there exists a continuous lifting $\tilde{\varphi}: A \rightarrow E$ of $\varphi$. The equation

$$
\gamma \cdot \tilde{\varphi}(a)=\tilde{\varphi}(a) \tilde{\alpha}_{a}(\gamma)
$$

valid for $a \in A$ and $\gamma \in \mathcal{I}_{a}$, determines a continuous representation $\alpha_{\eta, \tilde{\varphi}}: \mathcal{I} \rightarrow G$.

Lemma 3.1. Suppose that $\Gamma$ and $G$ are compact Lie groups and that $A$ is locally compact. If $\mathcal{I}$ is locally maximal, then the continuous representation $\alpha_{\eta, \tilde{\varphi}}$ is weakly locally maximal.

Proof. Let $a \in A$ and let $B$ be a compact neighbourhood of $a$. Since $\mathcal{I}$ is locally maximal, there exists an open set $U$, with $a \in U \subset B$ and a homotopy $\rho_{t}: U \rightarrow U$ such that $\rho_{0}(u)=u, \rho_{1}(u)=a$ and $\mathcal{I}_{u} \subset \mathcal{I}_{\rho_{t}(u)}$ for all $u \in U$. If $Z \subset A$, we denote $\hat{Z}=\pi^{-1}(Z)$; if $Y$ is a $\Gamma$-invariant subspace of $X$, we denote $E_{Y}=p^{-1}(Y)$. The latter is the total space of a split $\Gamma$-equivariant $G$-principal bundle $\eta_{Y}$ over $Y$.

By Proposition 2.1 and its proof, the space $\hat{B}$ is compact. Then $E_{\hat{B}}$ is compact and therefore totally regular. By [27], Proposition 8.10, the bundle $\eta_{\hat{B}}$ is then a locally trivial numerable $\Gamma$-equivariant $G$-principal bundle in the sense of [27], p. 58. The same then holds for its restriction $\eta_{\widehat{U}}$.

By Lemma 2.3 and its proof, the homotopy $\rho_{t}: U \rightarrow U$ is covered by a $\Gamma$ equivariant homotopy $\hat{\rho}_{r}: \widehat{U} \rightarrow \widehat{U}$ such that $\hat{\rho}_{0}=\mathrm{id}$ and $\hat{\rho}_{1}(\widehat{U})=\pi^{-1}(a)$. By [27], Theorem 8.15, the induced bundle $\hat{\rho}_{1}^{*} \eta_{\pi^{-1}(a)}$ is then isomorphic to $\eta_{\hat{U}}$. More precisely, let

$$
E_{1}:=\left\{(x, z) \in \widehat{U} \times E_{\pi^{-1}(a)} \mid \hat{\rho}_{1}(x)=p(z)\right\}
$$

be the total space of $\hat{\rho}_{1}^{*} \eta_{\pi^{-1}(a)}$. Then there is a $(\Gamma \times G)$-equivariant homeomorphism $\mu: E_{1} \rightarrow E_{\widehat{U}}$ which commutes with the projections onto $\widehat{U}$. By Lemma 2.3 one has $\varphi \circ \rho_{t}=\hat{\rho}_{t} \circ \varphi$; therefore, $(\varphi(u), \varphi(a)) \in E_{1}$ for all $u \in U$. This enables to define $\tilde{\varphi}^{\prime}: U \rightarrow E$ by $\tilde{\varphi}^{\prime}(u)=\mu(\varphi(u), \tilde{\varphi}(a))$. For $\gamma \in \mathcal{I}_{u} \subset \mathcal{I}_{a}$, we have

$$
\gamma \cdot \tilde{\varphi}^{\prime}(u)=\mu(\varphi(u), \gamma \tilde{\varphi}(a))=\mu\left(\varphi(u), \tilde{\varphi}(a) \alpha_{a}(\gamma)\right)=\mu(\varphi(u), \tilde{\varphi}(a)) \cdot \alpha_{a}(\gamma) .
$$


On the other hand, $\tilde{\varphi}$ and $\tilde{\varphi}^{\prime}$ are two liftings of $\varphi$ over $U$. Hence, there exists a continuous map $g: U \rightarrow G$ such that $\tilde{\varphi}^{\prime}(u)=\tilde{\varphi}(u) \cdot g(u)$ for all $u \in U$. Therefore

$$
\gamma \cdot \tilde{\varphi}^{\prime}(u)=\gamma \tilde{\varphi}(u) g(u)=\tilde{\varphi}(u) \alpha_{u}(\gamma) g(u)=\tilde{\varphi}^{\prime}(u) \cdot\left(g(u)^{-1} \alpha_{u}(\gamma) g(u)\right) .
$$

Comparing equations (2) with (3), we get that $\alpha_{u}(\gamma)=g(u) \alpha_{a}(\gamma) g(u)^{-1}$, which proves Lemma 3.1.

By Lemma 3.1, $\alpha_{\eta, \tilde{\varphi}}$ determines a class $\alpha_{\eta} \in \operatorname{Rep}^{G}(\mathcal{I})$. We check that $\alpha_{\eta}$ does not depend on the choice of $\tilde{\varphi}$ and depends only on the $\Gamma$-equivariant isomorphism class of $\eta$; details are as in the proof of [13], Lemma 3.2. This defines a map

$$
\Phi: \operatorname{SBun}_{\Gamma}^{G}(X) \rightarrow \operatorname{Rep}^{G}(\mathcal{I})
$$

called the isotropy representation.

§3C. The classification theorem. The following theorem corresponds to Theorem A of the introduction.

Theorem 3.2 (Classification). Let $(X, \pi, \varphi)$ be a split $\Gamma$-space over $A$ with isotropy groupoid I. Suppose that $A$ is locally compact, that $\mathcal{I}$ is locally maximal and that $\Gamma$ is a compact Lie group. Then, for any compact Lie group $G$, the isotropy representation $\Phi: \operatorname{SBun}_{\Gamma}^{G}(X) \rightarrow \operatorname{Rep}^{G}(\mathcal{I})$ is a bijection.

Proof. We first prove the surjectivity of $\boldsymbol{\Phi}$. By Proposition 2.1, we may assume that $(X, \pi, \varphi)=\left(Y_{\mathcal{I}}, \Pi, \phi\right)$. Recall that $Y_{\mathfrak{I}}=\Gamma \times_{\mathfrak{I}} A$.

Let $\beta: \mathcal{I} \rightarrow G$ be a continuous representation. Then $\mathcal{I}$ acts on the left on $A \times G$ by $\zeta \cdot(a, g)=(a, \beta(\zeta) g)$. Form the space $E_{\beta}=\Gamma \times \mathcal{I}(A \times G)$. The continuous map $p: E_{\beta} \rightarrow Y_{\mathcal{I}}$ given $p([\gamma,(a, g)])=[\gamma, a]$ coincides with the projection $E_{\beta} \rightarrow$ $E_{\beta} / G$ of $E_{\beta}$ to its orbit space for the obvious free right $G$-action on $E_{\beta}$. A lifting $\tilde{\phi}: A \rightarrow E_{\beta}$ of $\phi$ is given by $\tilde{\phi}(a)=[1,(a, 1)]$, where 1 denotes the unit elements. For $a \in A$ and $\gamma \in \mathcal{I}_{a}$, one has

$$
\gamma \cdot \tilde{\phi}(a)=\gamma \cdot[1,(a, 1)]=[\gamma,(a, 1)]=\left[1,\left(a, \beta_{a}(\gamma)\right]=\tilde{\phi}(a) \cdot \beta_{a}(\gamma) .\right.
$$

We now prove that $p$ admits local trivializations when $\beta$ is locally maximal. Let $a \in A$. Choose an open neighbourhood $U_{a}$ of $a$ such that $\mathcal{I}_{u}$ is a subgroup of $\mathcal{I}_{a}$ for all $u \in U_{a}$, together with a continuous map $g_{a}: U_{a} \rightarrow G$ such that $\beta_{u}(\gamma)=$ $g_{a}(u) \beta_{a}(\gamma) g_{a}(u)^{-1}$ for all $u \in U_{a}$ and all $\gamma \in \mathcal{I}_{u}$. This gives an open cover $\mathcal{U}=\left\{U_{a} \mid a \in A\right\}$ of $A$. Setting $\widehat{U}_{a}=\Pi^{-1}\left(U_{a}\right)$ gives rise to an open cover $\hat{U}=\left\{\widehat{U}_{a} \mid a \in A\right\}$ of $X$, indexed by $A$. Define $\tilde{f}_{a}: \Gamma \times U_{a} \times G \rightarrow \Gamma \times\{a\} \times G$ by $f_{a}(\gamma, u, g):=\left(\gamma, a, g_{a}(u) g\right)$. If $\delta \in \mathcal{I}_{u}$, we have

$$
\tilde{f}_{a}(\gamma \delta, u, g)=\left(\gamma \delta, a, g_{a}(u) g\right)=\left(\gamma, a, \beta_{a}(\delta) g_{a}(u) g\right)
$$


and

$$
\tilde{f}_{a}\left(\gamma, u, \beta_{u}(\delta) g\right)=\left(\gamma, a, g_{a}(u) \beta_{u}(\delta) g\right) .
$$

Since $\beta_{a}(\delta) g_{a}(u)=g_{a}(u) \beta_{u}(\delta)$, this shows that $\tilde{f}_{a}$ descends to a continuous $G$ equivariant map $f_{a}: p^{-1}\left(\hat{U}_{a}\right) \rightarrow p^{-1}\left(\pi^{-1}(a)\right)$. Passing to the quotient by $G$ gives rise to a commutative diagram

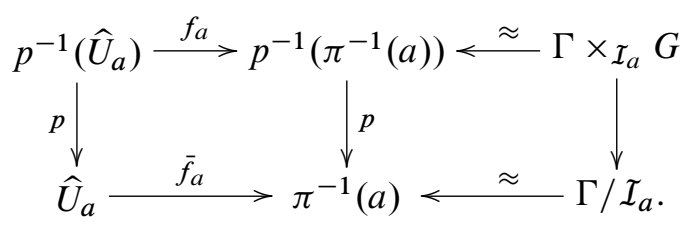

Since $\Gamma$ is a Lie group and $\mathcal{I}_{a}$ a closed subgroup, the projection $q_{a}: \Gamma \rightarrow \Gamma / \mathcal{I}_{a}$ admits local sections $\sigma_{V}: V \rightarrow \Gamma$ for each $V$ in some open covering $\mathcal{V}_{a}$ of $\Gamma / \mathcal{I}_{a}$ (see, e.g., [24], §7.5). We check that the formula

$$
\zeta_{V}(\gamma, g)=\beta_{a}\left(\sigma(q(\gamma))^{-1} \gamma\right) g
$$

defines a $G$-equivariant continuous map $\zeta_{V}: p^{-1}(V) \rightarrow G$, which gives rise to a trivialization over $V$ of $p: \Gamma \times \mathcal{I}_{a} G \rightarrow \Gamma / \mathcal{I}_{a}$. Therefore, $\zeta_{V} \circ f_{a}: p^{-1}\left(\bar{f}_{a}^{-1}(V)\right) \rightarrow$ $G$ is a $G$-equivariant continuous map giving rise to a trivialization over $f_{a}^{-1}(V)$ of $p: p^{-1}\left(\hat{U}_{a}\right) \rightarrow \widehat{U}_{a}$. This gives rise to a trivializing open cover $\mathcal{W}=\left\{\bar{f}_{a}^{-1}(V) \mid\right.$ $(a, V) \in \mathcal{A}\}$ of $X$, indexed by $\mathcal{A}=\left\{(b, V) \mid b \in A\right.$ and $\left.V \in \mathcal{V}_{b}\right\}$. We have proved thus the surjectivity of $\boldsymbol{\Phi}$.

We now prove the injectivity of $\boldsymbol{\Phi}$. Let $\eta:(E \stackrel{\bar{p}}{\rightarrow} X)$ be a split $\Gamma$-equivariant bundle with $\boldsymbol{\Phi}(\eta)=[\beta]$. A lifting $\bar{\varphi}: A \rightarrow E$ of $\varphi$ then produces a continuous representation $\bar{\beta}=\alpha_{\eta, \bar{\varphi}}$ with $[\bar{\beta}]=[\beta]$. There exists then a continuous map $\psi: A \rightarrow G$ such that $\beta(\zeta)=\psi(q(\zeta))^{-1} \bar{\beta}(\zeta) \psi(q(\zeta))$. The map $\tilde{\varphi}: A \rightarrow E$ given by $\tilde{\varphi}(a)=\bar{\varphi}(a) \cdot \psi(a)$ is then another lifting of $\varphi$ such that $\alpha_{\eta, \tilde{\varphi}}=\beta$. One checks that the correspondence $[\gamma,(a, g)] \mapsto \gamma \cdot \varphi(a) \cdot g$ defines a $(\Gamma \times G)$-equivariant continuous bijection $\widetilde{F}: E_{\beta} \rightarrow E$, covering the unique $\Gamma$-equivariant homeomorphism $F: Y_{\mathcal{I}} \rightarrow X$ such that $F \circ \varphi=\phi$, obtained in Proposition 2.1. Since $F$ is a homeomorphism, so is $\widetilde{F}$. Indeed, choose an open set $Z$ in $Y_{\mathcal{I}}$ such that $\xi_{\beta}$ is trivial over $Z$ and $\xi$ is trivial over $F(Z)$. Using trivializations, we can write $\widetilde{F}(z, g)=(F(z), \mu(z) g)$, where $\mu: Z \rightarrow G$ is a continuous map. Then $\widetilde{F}^{-1}$ has, over $F(Z)$, the form $\widetilde{F}^{-1}(y, h)=\left(F^{-1}(y), \mu\left(F^{-1}(y)\right)^{-1} h\right)$, which is continuous. We have thus proven that two split $\Gamma$-equivariant principal $G$-bundles $\eta$ and $\eta^{\prime}$ with $\boldsymbol{\Phi}(\eta)=\boldsymbol{\Phi}\left(\eta^{\prime}\right)$ are $\Gamma$-equivariantly isomorphic.

Remark 3.3. Recall that an open cover of a space is numerable if it admits a refinement by a locally finite partition of unity. In the proof of Theorem 3.2 , the covers $\mathcal{V}_{a}$ are numerable, since $\Gamma / \mathcal{I}_{a}$ are manifolds. Hence we can check that the trivializing cover $\mathcal{W}$ of $X$ is numerable if $\mathcal{U}$ is numerable. This observation will be used in Theorem 4.5. 
3.4. Non-split bundles and abelian structure group. Let $(X, \pi, \varphi)$ be a split $\Gamma$ space over $A$. One has the map $\varphi^{*}: \operatorname{Bun}_{\Gamma}^{G}(X) \rightarrow \operatorname{Bun}^{G}(A)$, sending $\xi$ to $\varphi^{*} \xi$. This map is surjective: if $\eta \in \operatorname{Bun}^{G}(A)$, then $\pi^{*} \eta$ admits a natural $\Gamma$-action, since $\pi$ is $\Gamma$-invariant, so $\pi^{*}$ is a section of $\varphi^{*}$. Theorem 3.2 computes the pre-image of the trivial bundle, which is $\operatorname{SBun}_{\Gamma}^{G}(X)$.

Let us now assume that $G$ is abelian. Recall that there is then a composition law " $\otimes$ " on $\operatorname{Bun}_{\Gamma}^{G}(X)$ which makes the latter an abelian group. If $\xi_{i}: E_{i} \stackrel{p_{i}}{\rightarrow} X$ $(i=1,2)$ are $\Gamma$-equivariant principal $G$-bundles, one defines $\xi_{1} \otimes \xi_{2}: E \stackrel{p_{i}}{\longrightarrow} X$ by first forming the pull-back

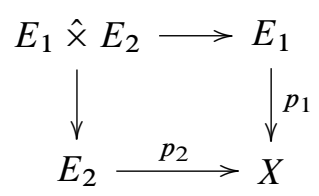

where the map $E_{1} \hat{\times} E_{2} \rightarrow X$ is a principal $G \times G$-bundle. Set $E=E_{1} \hat{\times}_{G} E_{2}$ (as $G$ is abelian, it acts on the left or on the right on $E_{i}$ ) and check that $\xi_{1} \otimes \xi_{2}$ is a principal $G$-bundle over $X$. The diagonal $\Gamma$-action on $E_{1} \hat{\times} E_{2}$ descends to a $\Gamma$-action on $E$, making $\xi_{1} \otimes \xi_{2}$ a $\Gamma$-equivariant principal $G$-bundle. When $G=S^{1}$, we can think of $\xi_{i}$ as $\Gamma$-equivariant complex line bundles over $X$, thus " $\otimes$ " becomes the standard tensor product. The map $\varphi^{*}: \operatorname{Bun}_{\Gamma}^{G}(X) \rightarrow \operatorname{Bun}^{G}(A)$ is a group-homomorphism.

Another special feature of the case $G$ abelian is that the isotropy representation is defined on $\operatorname{Bun}_{\Gamma}^{G}(X)$ : in equation (1), one can just use a local section $\tilde{\varphi}$ around $a \in A$, whose choice is irrelevant if $G$ is abelian. The set $\operatorname{Rep}^{G}(\mathcal{I})$ is an abelian group, by multiplication of the images, and $\boldsymbol{\Phi}: \operatorname{Bun}_{\Gamma}^{G}(X) \rightarrow \operatorname{Rep}^{G}(\mathcal{I})$ is a group homomorphism. Using Theorem 3.2, we get

Proposition 3.5. Let $(X, \pi, \varphi)$ be a split $\Gamma$-space over $A$ with isotropy groupoid $\mathcal{I}$. Suppose that $A$ is locally compact, that $\mathcal{I}$ is locally maximal and that $\Gamma$ is a compact Lie group. Then, for any compact abelian Lie group $G$, one has an isomorphism of abelian groups

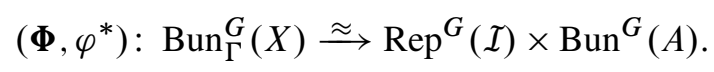

3.6. Functorial properties. Theorem 3.2 enjoys functorial properties which are contravariant in $(\Gamma, A)$ and covariant in $G$. For the contravariant ones, let $f: A^{\prime} \rightarrow A$ be a continuous map between locally compact spaces and $h: \Gamma^{\prime} \rightarrow \Gamma$ be a continuous homomorphism between compact Lie groups. Let $\mathcal{I}$ be a $(\Gamma, A)$-groupoid. Then

$$
\mathcal{I}^{\prime}:=(h, f)^{*} \mathcal{I}:=\left\{\left(\gamma^{\prime}, a^{\prime}\right) \in \Gamma^{\prime} \times A^{\prime} \mid h\left(\gamma^{\prime}\right) \in \mathcal{I}_{f\left(a^{\prime}\right)}\right\}
$$

is a $\left(\Gamma^{\prime}, A^{\prime}\right)$-groupoid, with $\tilde{I}_{a^{\prime}}^{\prime}=h^{-1}\left(\tilde{I}_{f\left(a^{\prime}\right)}\right) \times\left\{a^{\prime}\right\}$. One has the continuous map

$$
\Gamma^{\prime} \times \mathcal{I}^{\prime} A^{\prime} \stackrel{(h, f)}{\longrightarrow} \Gamma \times{ }_{\mathcal{I}} A .
$$


Therefore, if $\mathcal{I}$ and $\mathcal{I}^{\prime}$ are locally maximal, Proposition 2.1 together with equation (4) implies the following: if $(X, \pi, \varphi)$ and $\left(X^{\prime}, \pi^{\prime}, \varphi^{\prime}\right)$ are the split spaces with isotropy groupoids $\mathcal{I}$ and $\mathcal{I}^{\prime}$, there is a unique map $F=F_{h, f}: X^{\prime} \rightarrow X$ such that $F\left(\gamma^{\prime} x^{\prime}\right)=$ $h\left(\gamma^{\prime}\right) F\left(x^{\prime}\right), \pi \circ F=f \circ \pi^{\prime}$ and $\varphi \circ f=F \circ \varphi^{\prime}$. Let $\eta$ be a split $\Gamma$-equivariant principal $G$-bundle over $X$. Using Theorem 3.2, one checks that $\eta^{\prime}:=F^{*} \eta$ is a split $\Gamma^{\prime}$-equivariant principal $G$-bundle over $X^{\prime}$ and that the isotropy representations $\alpha^{\prime} \in \operatorname{Rep}^{G}\left(I^{\prime}\right)$ and $\alpha \in \operatorname{Rep}^{G}(\mathcal{I})$ satisfy $\alpha^{\prime}=h^{*} \alpha$, where $h^{*} \alpha=\alpha \circ h$. Therefore, one gets a commutative diagram

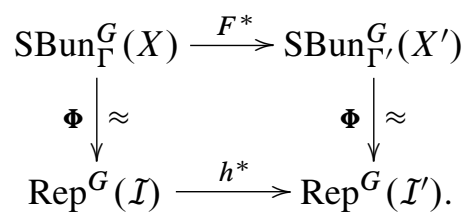

As for the covariant functoriality in $G$, let $\mu: G \rightarrow G^{\prime}$ be a continuous homomorphism between compact Lie groups. If $\eta:(E \rightarrow X)$ is a split $\Gamma$-equivariant principal $G$-bundle over $X$, one checks that $\mu_{*} \eta:\left(E \times{ }_{\mu} G^{\prime} \rightarrow X\right)$ is a split $\Gamma$-equivariant principal $G$-bundle with isotropy representation $\mu_{*} \alpha=\mu \circ \alpha$. One gets a commutative diagram

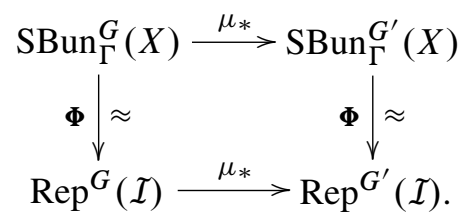

In particular, let $G=G^{\prime} \times G^{\prime \prime}$ and let $p^{\prime}$ and $p^{\prime \prime}$ be the two projections. Diagram (6) becomes

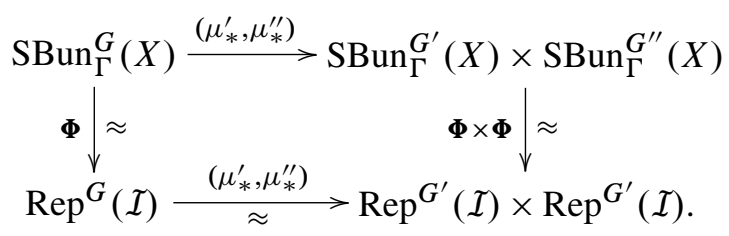

Diagram (7) then shows that the map

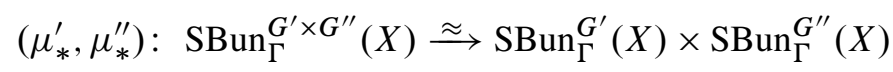

is a bijection.

\section{Cellular groupoids - examples}

\$ 4A. Cellular groupoids. Let $A$ be a CW-complex filtered by its skeleta $A^{(n)}$. We denote by $\Lambda=\Lambda(A)$ the set of cells of $A$. The dimension of a cell $e \in \Lambda$ is denoted 
by $d(e)$ and we set $\Lambda_{n}=\{e \in \Lambda \mid d(e)=n\}$. For each $e \in \Lambda$, there exists a characteristic map $\sigma_{e}:\left(\mathbb{D}^{d(e)}, \mathbb{S}^{d(e)-1}\right) \rightarrow\left(A^{(d(e))}, A^{(d(e)-1)}\right)$, and $\sigma_{e}$ restricted to the interior of $\mathbb{D}^{d(e)}$ is an embedding whose image is denoted by $|e|$. For $a \in A$, we denote by $e(a) \in \Lambda$ the cell $e$ of smallest dimension such that $a \in \sigma(e)$. The set $\Lambda$ is partially ordered: $f^{\prime} \leq f$ if $f^{\prime}$ is a face of $f$, which means that there exists $x \in \mathbb{S}^{d(f)-1}$ such that $e\left(\sigma_{f^{\prime}}(x)\right)=f$.

Let $\Gamma$ be a topological group and $A$ be a $C W$-complex. A $(\Gamma, A)$-groupoid $\mathcal{I}$ is called cellular if it is locally maximal and if $\tilde{\mathcal{I}}_{a}=\tilde{\mathcal{I}}_{b}$ when $e(a)=e(b)$. We write $\tilde{I}^{(n)}$ for the restriction of $\mathcal{I}$ over $A^{(n)}$. Recall that $\mathcal{I}_{a}=\tilde{\mathcal{I}}_{a} \times\{a\}$ where $\tilde{\mathcal{I}}_{a} \in \operatorname{Gr}(\Gamma)$, the poset of closed subgroups of $\Gamma$. One can then define a map $\tilde{I}: \Lambda(A) \rightarrow \operatorname{Gr}(\Gamma)$ by $\tilde{\mathcal{I}}(e)=\tilde{\mathcal{I}}_{a}$ for $a$ with $e(a)=e$. The local maximality of $\mathcal{I}$ implies that $\tilde{\mathcal{I}}(e) \subset \tilde{\mathcal{I}}(f)$ when $f \leq e$. Thus, $\tilde{\mathcal{I}}$ is a contravariant functor from the poset $\Lambda(A)$ to the poset $\operatorname{Gr}(\Gamma)$. A cellular groupoid is a combinatorial construction.

Lemma 4.1. The correspondence $\mathcal{I} \rightarrow \tilde{I}$ is a bijection between the set of cellular groupoids whose object-space is $A$ and the set of contravariant functors from $\Lambda(A)$ to $\operatorname{Gr}(\Gamma)$.

Proof. The correspondence is clearly injective. For the surjectivity, let $\mathcal{F}: e \mapsto \mathcal{F}_{e}$ be a contravariant functor from $\Lambda(A)$ to $\operatorname{Gr}(\Gamma)$. By induction on $n$, we shall construct $\mathcal{I}^{(n)}$, giving rise to a $(\Gamma, A)$-groupoid $\mathcal{I}$, with $\tilde{\mathcal{I}}=\mathscr{F}$ and then check that $\mathcal{I}$ is locally maximal. Define $\mathcal{I}^{(0)}=\bigsqcup_{v \in \Lambda_{0}(A)} \mathscr{F}_{v} \times\{v\}$. Suppose that $\mathcal{I}^{(n-1)}$ is constructed. The $n$-skeleton $A^{(n)}$ of $A$ is obtained as the quotient space

$$
A^{(n)}=\left(\underset{e \in \Lambda_{n}(A)}{\left.\mathbb{D}_{e}\right) \amalg A^{(n-1)} /\left\{x \sim \sigma_{e}(x) \mid x \in \mathbb{S}_{e}\right\},}\right.
$$

where $\left(\mathbb{D}_{e}, \mathbb{S}_{e}\right)$ is a copy of $\left(\mathbb{D}^{n}, \mathbb{S}^{n-1}\right)$ and $\sigma_{e}: \mathbb{D}_{e} \rightarrow A$ is a characteristic map for the cell $e$. We then define

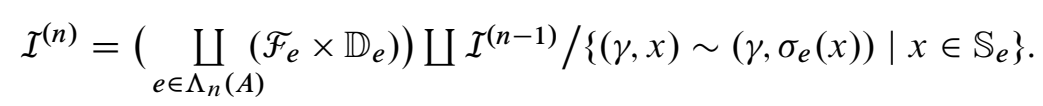

The equivalence relation $\sim$ makes sense since, for $x \in \mathbb{S}_{e}$, one has $\mathscr{F}_{e} \subset \tilde{\mathcal{I}}_{\mu_{e}(x)}^{(n-1)}$. Clearly, $\tilde{\mathcal{I}}=\mathscr{F}$. Now each $a \in A$ admits a fundamental system of open neighbourhoods $U$ of $a$ such that $e(a) \leq e(u)$ for all $u \in U$. One can also require that $U$ admits a homotopy $\rho_{t}: U \rightarrow U$ such that $\rho_{0}=\mathrm{id}, \rho_{1}(U)=\{a\}$ and $e\left(\rho_{t}(u)\right)=e(u)$ for $t<1$ (see [21], Theorem 6.1 and its proof, or proof of Lemma 4.4 below). Therefore, $\mathcal{I}$ is locally maximal.

The notation $\tilde{\mathcal{I}}$ was introduced in order to state and prove Lemma 4.1 properly. In future occurrences we shall write $\mathcal{I}(e)$ instead of $\tilde{I}(e)$.

Remark 4.2. Let $(X, \pi, \varphi)$ be a split $\Gamma$-space over a CW-complex $A$, with a cellular isotropy groupoid $\mathcal{I}$. Then $X$ is provided with a $\Gamma$-equivariant $C W$-complex structure 
(see, e.g., [27], Chapter 2) with $\Gamma$-cells indexed by $\Lambda(A)$. If $\sigma_{e}: \mathbb{D}^{d(e)} \rightarrow A$ is a characteristic map for $e \in \Lambda(A)$, then $\tilde{\sigma}_{e}: \Gamma / \mathcal{I}(e) \times \mathbb{D}^{d(e)} \rightarrow X$, defined by $\tilde{\sigma}(\gamma, a)=\gamma \varphi(a)$, is a characteristic map for the $\Gamma$-cell of $X$ corresponding to $e$.

On the other hand, let $X$ be a $\Gamma$-CW-complex and $A=\Gamma / X$ be its orbit space with the induced $\mathrm{CW}$-structure. Suppose that there exists a section $\varphi: A \rightarrow X$ of the projection $\pi: X \rightarrow A$, so that the isotropy groupoid $\mathcal{I}$ is weakly locally maximal. Then $\mathcal{I}$ is cellular, since $\mathcal{I}_{a}$ is constant on the interior of each cell. We call $X$ a split $\Gamma$-CW-complex over $A$.

However, one has the following example of a split $\Gamma$-space over a $\mathrm{CW}$-complex admitting no splitting for which the isotropy groupoid is cellular. Let $X=([0,1] \times$ $\left.S^{2}\right) /\{(1, x) \sim(0,-x)\}$, the mapping cylinder of the antipodal map of $S^{2}$, endowed with the natural action of $\Gamma=\mathrm{SO}(3)$. Then $A=[0,1] /\{0 \sim 1\} \approx S^{1}$. Any splitting is of the form $\varphi(t)=(t, f(t))$ with $\lim _{t \rightarrow 0} f(t)=-\lim _{t \rightarrow 1} f(t)$. Thus, $f(t)$ is not constant and $\mathcal{I}$ is not weakly locally maximal. Observe that $X$ is a smooth closed 3-manifold and that the $\mathrm{SO}(3)$-action is smooth with cohomogeneity one.

For cellular groupoids we have a stronger version of Proposition 2.1, which applies to any topological group $\Gamma$.

Proposition 4.3 (Reconstruction II). Let $\Gamma$ be a topological group, and $A$ be a CWcomplex. Given a cellular $(\Gamma, A)$-groupoid $\mathcal{I}$, there is a unique split $\Gamma$-CW-complex over A with isotropy groupoid $\mathcal{I}$.

Proof. The space $Y_{\mathcal{I}}$ is a split $\Gamma$-CW-complex over $A$. Suppose that $(X, \pi, \varphi)$ is another split $\Gamma$-CW-complex with isotropy groupoid $\mathcal{I}$. As in the proof of Proposition 2.1, the map $\widetilde{F}: \Gamma \times A \rightarrow X$ defined by $\widetilde{F}(\gamma, a)=\gamma \cdot \varphi(a)$ descends to give a continuous $\Gamma$-equivariant bijection $F: Y_{\mathcal{I}} \rightarrow X$. For each cell $e \in \Lambda_{n}(Y)$ with characteristic map $\sigma_{e}: \mathbb{D}^{n} \rightarrow A$, there is a commutative diagram

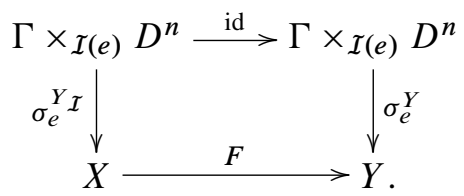

Therefore $F$ is an open map and hence a homeomorphism.

Let $\Gamma$ be a topological group and $A$ be a $C W$-complex. A $(\Gamma, A)$-groupoid $\mathcal{I}$ such that $\mathcal{I}_{a}$ is a compact Lie group for all $a \in A$ is called proper. When $\Gamma$ is itself a Lie group, this is equivalent to saying that the $\Gamma$-action on the corresponding split $\Gamma$-CW-complex with isotropy groupoid $\mathcal{I}$ is proper; see [19], Theorem 1.23. We have a classification theorem for equivariant bundles over split $\Gamma$-spaces with proper isotropy groupoids in Theorem 4.5. First we give a version of Lemma 3.1. 
Lemma 4.4. Let $\Gamma$ be a topological group, and $A$ be a $C W$-complex. Let I be a proper $(\Gamma, A)$-groupoid. Then any continuous representation of $\mathcal{I}$ to a compact Lie group $G$ is weakly locally maximal.

Proof. Let $\alpha: \mathcal{I} \rightarrow G$ be a continuous representation. Let $a \in A$. We shall construct a pair $(U, g)$, where $U$ is an open set of $A$, such that $\mathcal{I}_{u}$ is a subgroup of $\mathcal{I}_{a}$ for each $u \in U$, and $g: U \rightarrow G$ is a continuous map satisfying $\alpha_{u}(\gamma)=g(u) \alpha_{a}(\gamma) g(u)^{-1}$ for all $u \in U$ and all $\gamma \in \mathcal{I}_{u}$. Call the pair $(U, g)$ an a-straightening of $\alpha$ in $A$. The final open set $U$ will be a neighbourhood of $a$, but the definition of an $a$-straightening does not use that $a \in U$, just that the element $g(a) \in G$ is defined. An $a$-straightening is equivalent to the data of a sequence $\left(U^{(d)}, g_{d}\right)$ of $a$-straightenings of $\alpha$ in $A^{(d)}$, such that $U^{(d+1)} \cap A^{(d)}=U^{(d)}$ and $g_{d+1} \mid U^{(d)}=g_{d}$.

We construct $\left(U^{(d)}, g_{d}\right)$ by induction on $d$, setting $U_{d}=\emptyset$ if $d<d(e(a))$. If $d(e(a))=0$, then we set $U^{(0)}=\{a\}$ and $g_{0}(a)=1$. If $d(e(a))>0$, then there exists a neighbourhood $U^{d(e(a))}$ of $a$ in $e(a)$ with a pointed homeomorphism $\left(U^{(d(e(a)))}, a\right) \underset{\approx}{\approx}\left([-1,1]^{d(e(a))}, 0\right)$. The existence of $g_{d(e(a))}$ is guaranteed by Lemma 1.2. Suppose that an $a$-straightening $\left(U^{(d)}, g_{d}\right)$ of $\alpha$ in $A^{(d)}$ is constructed, with $d \geq d(e(a))$ and $a \in U_{d}$. For $e \in \Lambda_{d+1}(A)$, let $\sigma_{e}:\left(\mathbb{D}_{e}^{d+1}, \mathbb{S}_{e}^{d}\right) \rightarrow$ $\left(A^{(d+1)}, A^{(d)}\right)$ be a characteristic map for the cell $e$. Let $V_{e}$ be the open set of $\mathbb{S}_{e}^{d}$ defined by $V_{e}=\sigma_{e}^{-1}\left(U^{(d)}\right)$. Let $W_{e}$ be the open set of $\mathbb{D}_{e}^{d+1}$ defined by $W_{e}=$ $\left\{t x \mid x \in V_{e}\right.$ and $\left.t \in(0,1]\right\}$. The correspondence $u \mapsto \alpha_{\sigma_{e}(u)} \in \operatorname{Hom}(\mathcal{I}(e), G)$ is a continuous representation $\alpha_{e}$ of the $\left(\Gamma, \mathbb{D}_{e}^{n+1}\right)$-groupoid $\mathcal{I}(e) \times \mathbb{D}_{e}^{n+1}$. The pair $\left(V_{e}, g_{d} \circ \sigma_{e}\right)$ is a $a$-straightening of $\alpha_{e}$ in $\mathbb{S}_{e}^{n}$. As $W_{e}$ is homeomorphic to $V_{e} \times[0,1]$, this $a$-straightening extends to a $a$-straightening $\left(W_{e}, g_{e}\right)$ of $\alpha_{e}$ in $\mathbb{D}_{e}^{n+1}$. The family $g_{e}$ defines a map $g_{d+1}: U^{(n+1)} \rightarrow G$, where $U^{(n+1)}=\bigcup_{e \in \Lambda_{n+1}(A)} W_{e}$, giving rise to the $a$-straightening of $\alpha$ in $A^{(n+1)}$.

By Lemma 4.4, the isotropy representation $\Phi: \operatorname{SBun}_{\Gamma}^{G}(X) \rightarrow \operatorname{Rep}^{G}(\mathcal{I})$ is defined, as in Section $\S 3 \mathrm{~B}$. The classification theorem for split bundles over a split $\Gamma$-CW-complex with proper isotropy groupoid takes the following form.

Theorem 4.5 (Classification II). Let $\Gamma$ be a Lie group, and A be a CW-complex. Let I be a proper $(\Gamma, A)$-groupoid. Let $(X, \pi, \varphi)$ be a split $\Gamma$-CW-complex over $A$ with isotropy groupoid $\mathcal{I}$. Then, for any compact Lie group $G$, the isotropy representation $\Phi: \operatorname{SBun}_{\Gamma}^{G}(X) \rightarrow \operatorname{Rep}^{G}(\mathcal{I})$ is a bijection. Moreover, any split bundle over $X$ is numerable.

Proof. The proof of Theorem 4.5 is the same as that of Theorem 3.2, using Lemma 4.4 instead of Lemma 3.1 and Proposition 4.3 instead of Proposition 2.1. Being a CWcomplex, $A$ is paracompact, so each open cover is numerable. The last assertion of Theorem 4.5 comes from Remark 3.3.

Remark 4.6. The assumption that $\Gamma$ is a Lie group is only used to ensure that the quotient projection $q_{a}: \Gamma \rightarrow \Gamma / \mathcal{I}_{a}$ is a (numerable) principal bundle. If we do 
not care about numerability, the existence of local cross-sections of $q_{a}$ holds more generally (see [22] and [23]).

As in Proposition 3.5, Theorem 4.5 extends to a classification of all $\Gamma$-equivariant $G$-bundles if $G$ is abelian. More precisely:

Proposition 4.7. Let $\mathcal{I}$ be a proper $(\Gamma, A)$-groupoid for a Lie group $\Gamma$. Let $(X, \pi, \varphi)$ be a split $\Gamma$-CW-complex over $A$ with isotropy groupoid $\mathcal{I}$. Then, for any compact abelian Lie group $G$, one has an isomorphism of abelian groups

$$
\left(\Phi, \varphi^{*}\right): \operatorname{Bun}_{\Gamma}^{G}(X) \stackrel{\approx}{\longrightarrow} \operatorname{Rep}^{G}(\mathcal{I}) \times \operatorname{Bun}^{G}(A) .
$$

Moreover, any principal $\Gamma$-equivariant $G$-bundle over $X$ is numerable.

Proof. The proof Proposition 4.7 is the same as that of Proposition 3.5, using Theorem 4.5 instead of Theorem 3.2. For the numerability, observe that the inverse of the bijection $\left(\boldsymbol{\Phi}, \varphi^{*}\right)$ is given by $\left(\boldsymbol{\Phi}, \varphi^{*}\right)^{-1}(\xi, \eta)=\xi \otimes \pi^{*} \eta$. By Theorem 3.2, $\xi$ is numerable. Since $A$ is a CW-complex, $\eta$ is numerable and thus $\left(\boldsymbol{\Phi}, \varphi^{*}\right)^{-1}(\xi, \eta)$ is numerable. Hence, any $\Gamma$-equivariant principal $G$-bundle over $X$ is numerable.

\section{§ 4B. Examples}

4.8. Generalised toric manifolds of real dimension $2 m$, in the sense of [6], are split $\mathbb{T}$-spaces where $\mathbb{T}$ is an $m$-dimensional torus. The orbit space $A$ is a simple polytope and the section $\varphi$ is given in [6], Lemma 1.4. This includes symplectic toric manifolds, see, e.g., [11], where $\pi: X \rightarrow A$ is the moment map and $A \subset \operatorname{Lie}(\mathbb{T})^{*}$ the moment polytope. Our reconstruction Proposition 2.1 is the topological content of Delzant's theorem [11], Theorem 1.8, or [6], Proposition 1.7.

4.9. When $\Gamma$ is discrete, the "strata preserving actions with strict fundamental domain" of [4], Chapter II.12, are generalizations of split $\Gamma$-spaces with a cellular isotropy groupoid. Several examples are given in [4], Chapter II.12.9.

Several of the examples below involve $(\Gamma, A)$-groupoids where $A=\Delta^{m}$ is the standard $m$-simplex in $\mathbb{R}^{m+1}$ :

$$
\Delta^{m}=\left\{\left(t_{0}, \ldots, t_{m}\right) \in \mathbb{R}^{m+1} \mid t_{i} \geq 0 \text { and } \sum_{i=0}^{m} t_{i}=1\right\} .
$$

We use the standard simplicial structure on $\Delta^{m}$, with $\Lambda_{k}\left(\Delta^{m}\right)$ being the set of all subsets of $\{0, \ldots, m\}$ containing $k+1$ elements. When $m=1,2$, we use special notations illustrated by the following pictures. 
0

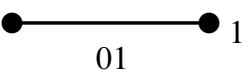

0

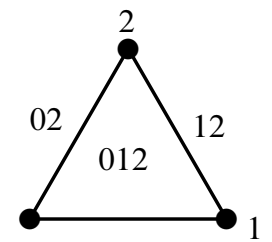

01

4.10. Let $\mathbb{T}=\left(S^{1}\right)^{m+1}$. We define a cellular $(\mathbb{T}, A)$-groupoid $\mathcal{I}$ with $A=\Delta^{m}$ by

$$
\mathcal{I}(e)=\left\{\left(\gamma_{0}, \ldots, \gamma_{m}\right) \mid \gamma_{i}=1 \text { if } i \in e\right\} .
$$

A model $(X, \pi, \varphi)$ for the split $\mathbb{T}$-space with isotropy groupoid $\mathcal{I}$ is given by $X=$ $S^{2 m+1} \subset \mathbb{C}^{m+1}$ with the $\mathbb{T}$-action $\left(\gamma_{0}, \ldots, \gamma_{m}\right) \cdot\left(z_{0}, \ldots, z_{m}\right)=\left(\gamma_{0} z_{0}, \ldots, \gamma_{m} z_{m}\right)$. The map $\pi$ and $\varphi$ may be chosen as

$$
\begin{aligned}
\pi\left(z_{0}, \ldots, z_{m}\right) & =\left(\left|z_{0}\right|^{2}, \ldots,\left|z_{m}\right|^{2}\right), \\
\varphi\left(t_{0}, \ldots, t_{m}\right) & =\left(\sqrt{t_{0}}, \ldots, \sqrt{t_{m}}\right) .
\end{aligned}
$$

More generally, let $\mathbb{T}$ be any torus and let $\chi_{0}, \ldots \chi_{m} \in \operatorname{Hom}\left(\mathbb{T}, S^{1}\right)$. Define a cellular $(\mathbb{T}, A)$-groupoid $\mathcal{I}$ with $A=\Delta^{m}$ by $\mathcal{I}(e)=\bigcap_{j \in e} \operatorname{ker} \chi_{j}$. A model for the split $\mathbb{T}$-space with isotropy groupoid $\mathcal{I}$ is again given by $\left(S^{2 m-+}, \pi, \varphi\right)$, where $\pi$ and $\varphi$ are defined by equations (9) and where the $\mathbb{T}$-action on $S^{2 m+1}$ is

$$
\gamma \cdot\left(z_{0}, \ldots, z_{m}\right)=\left(\chi_{0}(\gamma) z_{1}, \ldots, \chi_{m}(\gamma) z_{m}\right) .
$$

4.11. Let $\mathcal{I}$ be a $(\Gamma, A)$-groupoid and let $\Gamma_{0}$ be a closed subgroup of $\Gamma$. A $(\Gamma, A)$ groupoid $\mathcal{I}_{0}$ is then defined on $A$ by $\mathcal{I}_{0}(e)$ be the subgroup generated by $\mathcal{I}(e) \cup \Gamma_{0}$. If $(X, \pi, \varphi)$ is the split $\Gamma$-space over $A$ with isotropy groupoid $\mathcal{I}$, then that with isotropy groupoid $\mathcal{I}\left(\Gamma_{0}\right)$ is $\left(\Gamma_{0} \backslash X, \pi^{0}, \varphi^{0}\right)$, where $\pi^{0}$ is induced by $\pi$ and $\varphi^{0}$ is $\varphi$ composed with the projection $X \rightarrow \Gamma_{0} \backslash X$. For instance, if we take $\Gamma_{0}$ to be the diagonal $S^{1}$ in Example 4.10, we get a split $\left(S^{1}\right)^{m+1}$-structures on the complex projective space $\mathbb{C} P^{m}$.

4.12. Let $\Gamma=\operatorname{SO}(n+1)$. We see $\mathrm{SO}(n)$ as the subgroup of $\Gamma$ leaving the last coordinate fixed. Consider the $(\Gamma, A)$-groupoid with $A=[-1,1]$, defined by $\mathcal{I}_{ \pm 1}=$ $\Gamma$ and $\mathcal{I}_{(-1,1)}=\operatorname{SO}(n)$. The split $\Gamma$-space with isotropy groupoid $\mathcal{I}$ is $\left(S^{n}, \pi, \varphi\right)$ with $\pi\left(x_{1}, \ldots, x_{n+1}\right)=x_{n+1}(\varphi$ may be defined using a meridian). The classification of split $\Gamma$-equivariant $G$-bundles over $S^{n}$ has been studied in [13].

4.13. Let $X$ be a $\Gamma$-CW-complex $X$ so that the orbit space, with its induced $\mathrm{CW}$ structure, is a segment ( say $\Delta^{1}$ ). This is one type of cohomogeneity one action. There are then subgroups $\Gamma_{0}, \Gamma_{1}, \Gamma_{01}$ of $\Gamma$ so that $X$ is $\Gamma$-equivariantly homeomorphic to $\Gamma / \Gamma_{01} \times[0,1]$ glued to $\Gamma / \Gamma_{0} \times\{0\}$ and $\Gamma / \Gamma_{1} \times\{1\}$ by equivariant maps. Sending $t$ to $([e], t) \in \Gamma / \Gamma_{01} \times[0,1]$ produces a splitting $\varphi$ with a cellular isotropy groupoid $\mathcal{I}$, 
satisfying $\mathcal{I}_{01}=\Gamma_{01}, \mathcal{I}_{0}=\Gamma_{0}$ and $\mathcal{I}_{1}=\Gamma_{1}$. The space $X$ is then a split $\Gamma$-space with isotropy groupoid $\mathcal{I}$. If $\Gamma$ is a compact Lie group, one checks that $X$ has a natural smooth manifold structure for which the action is smooth. For more details and references on cohomogeneity one action, see [13], $\S 8$, where $\Gamma$-equivariant $G$ bundles over such $\Gamma$-spaces are classified (they are all split).

4.14. Let $\mathbb{T}$ be any torus and let $\chi$ be a non-trivial element in $\operatorname{Hom}\left(\mathbb{T}, S^{1}\right)$. Define a cellular $\left(\mathbb{T}, A\right.$ )-groupoid $\mathcal{I}$ with $A=\Delta^{1}$ by $\mathcal{I}_{0}=\mathcal{I}_{1}=\mathbb{T}$ and $\mathcal{I}_{01}=\operatorname{ker} \chi$. The split $\mathbb{T}$-space with isotropy groupoid $\mathcal{I}$ is $\left(\mathbb{C} P^{1}, \pi, \varphi\right)$, where $\pi\left(\left[z_{0}: z_{1}\right]\right)=$ $\left(\left|z_{0}\right|^{2},\left|z_{1}\right|^{2}\right), \varphi\left(t_{0}, t_{1}\right)=\left[\sqrt{t_{0}}: \sqrt{t_{1}}\right]$ and the $\mathbb{T}$-action is given by $\gamma\left[x_{0}: x_{1}\right]=$ $\left[\chi(\gamma) x_{0}: x_{1}\right]$. We denote this split $\mathbb{T}$-space by $\mathbb{C} P^{1}(\chi)$.

\section{Cellular representations - computations of $\operatorname{Rep}^{G}(\mathcal{I})$}

§5A. Cellular representations. Let $\mathcal{I}$ be a $(\Gamma, A)$-groupoid. A representation $\beta: \mathcal{I} \rightarrow G$ is called cellular if $\beta_{a}=\beta_{b}$ when $e(a)=e(b)$. For each $e \in \Lambda(A)$, this thus defines $\beta_{e} \in \operatorname{Hom}(\mathcal{I}(e), G)$, with the face compatibility conditions $\beta_{e}=$ $\beta_{f} \mid \mathcal{I}(e)$ whenever $f \leq e$. Two cellular representations $\alpha$ and $\beta$ are called conjugate if there exists $g \in G$ such that $\beta(\gamma)=g^{-1} \alpha(\gamma) g$ for all $\gamma \in \mathcal{I}_{a}$ and all $a \in A$. Denote by $\operatorname{Rep}_{\text {cell }}^{G}(\mathcal{I})$ the set of conjugacy classes of cellular representations of $\mathcal{I}$ into $G$.

To a cellular representation $\alpha: \mathcal{I} \rightarrow G$ and a cell $e$ of $A$, one can associate its conjugacy class $\left[\alpha_{e}\right] \in \overline{\operatorname{Hom}}(\mathcal{I}(e), G)$. This gives rise to a map

$$
\kappa: \operatorname{Rep}_{\text {cell }}^{G}(\mathcal{I}) \rightarrow \prod_{e \in \Lambda(A)} \overline{\operatorname{Hom}}(\mathcal{I}(e), G) .
$$

If an element $\left(b_{e}\right)$ of this product is in the image of $\kappa$, it must satisfy the face compatibility conditions, that is the equation $b_{e}=b_{f} \mid \mathcal{I}(e)$ holds in $\operatorname{Hom}(\mathcal{I}(e), G)$ whenever $f \leq e$. We then define

$$
\overline{\operatorname{Rep}}_{\text {cell }}^{G}(\mathcal{I})=\left\{\left(b_{e}\right) \in \prod_{e \in \Lambda(A)} \overline{\operatorname{Hom}}(\mathcal{I}(e), G)\left|b_{e}=b_{f}\right| \mathcal{I}(e) \text { if } f \leq e\right\}
$$

and see $\kappa$ as a map $\kappa: \operatorname{Rep}_{\text {cell }}^{G}(\mathcal{I}) \rightarrow \overline{\operatorname{Rep}}_{\text {cell }}^{G}(\mathcal{I})$. When $\mathcal{I}$ is a proper $(\Gamma, A)$-groupoid, the map $\kappa$ sits in a commutative diagram

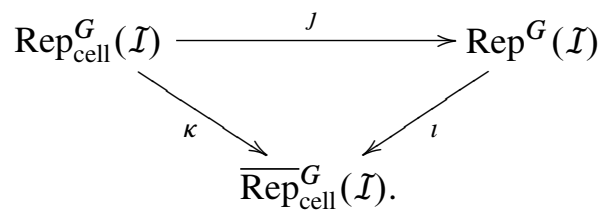

The map $J$ is obvious, since a cellular representation is a representation which is clearly weakly locally maximal. To define $\imath(\beta)_{e}$ for $e \in \Lambda(A)$, we choose $a \in A$ 
with $e(a)=e$ and set $t(\beta)_{e}=\left[\beta_{a}\right]$. Since cells are connected, $t$ is well defined by Lemma 1.1. Although none of these maps is either surjective or injective in general, diagram (10) is the source of all our information about $\operatorname{Rep}^{G}(\mathcal{I})$ so far.

One useful method for computing $\operatorname{Rep}_{\text {cell }}^{G}(\mathcal{I})$ and $\overline{\operatorname{Rep}}_{\text {cell }}^{G}(\mathcal{I})$ is to restrict representations of $\mathcal{I}$ to skeleta of $A$. This yields restriction maps $\operatorname{res}_{k}: \operatorname{Rep}_{\text {cell }}^{G}(\mathcal{I}) \rightarrow$ $\operatorname{Rep}_{\text {cell }}^{G}\left(\mathcal{I}^{(k)}\right)$ and $\operatorname{res}_{k}: \overline{\operatorname{Rep}}_{\text {cell }}^{G}(\mathcal{I}) \rightarrow \overline{\operatorname{Rep}}_{\text {cell }}^{G}\left(I^{(k)}\right)$. Recall that a CW-complex $A$ is regular if each cell $e$ admits a characteristic map $\sigma_{e}: \mathbb{D}^{d(e)} \rightarrow A$ that is an embedding, sending $\mathbb{S}^{d(e)-1}$ onto a subcomplex of $A^{(d(e)-1)}$. We set $\|e\|=\sigma_{e}\left(\mathbb{D}^{d(e)}\right)$, the closure of $|e|$. To simplify the notations, we write $\partial e$ instead of $\partial\|e\|$ for the boundary of $\|e\|$.

Proposition 5.1. Let I be a $(\Gamma, A)$-groupoid for $\Gamma$ a topological group. Assume that $A$ is a regular $C W$-complex. Then, for any topological group $G$, one has

(a) $\operatorname{res}_{0}: \operatorname{Rep}_{\text {cell }}^{G}(\mathcal{I}) \rightarrow \operatorname{Rep}_{\text {cell }}^{G}\left(\mathcal{I}^{(0)}\right)$ and $\operatorname{res}_{0}: \overline{\operatorname{Rep}}_{\text {cell }}^{G}(\mathcal{I}) \rightarrow \overline{\operatorname{Rep}}_{\text {cell }}^{G}\left(\mathcal{I}^{(0)}\right)$ are injective;

(b) $\operatorname{res}_{1}: \operatorname{Rep}_{\text {cell }}^{G}(\mathcal{I}) \rightarrow \operatorname{Rep}_{\text {cell }}^{G}\left(\mathcal{I}^{(1)}\right)$ and $\operatorname{res}_{1}: \overline{\operatorname{Rep}}_{\text {cell }}^{G}(\mathcal{I}) \rightarrow \overline{\operatorname{Rep}}_{\text {cell }}^{G}\left(\mathcal{I}^{(0)}\right)$ are bijective.

Proof. Let $\alpha \in \operatorname{Rep}_{\text {cell }}^{G}(\mathcal{I})$ (the proof for $\overline{\operatorname{Rep}}_{\text {cell }}^{G}(\mathcal{I})$ is the same). As $A$ is regular, each cell of $A$ has a face which is a vertex. Therefore, $\operatorname{res}_{0}(\alpha)$ determines $\alpha$ which proves (a) and the injectivity part of (b).

For the surjectivity in (b), it is enough to prove that the restriction map $\operatorname{Rep}_{\text {cell }}^{G}\left(\mathcal{I}^{(n)}\right) \rightarrow \operatorname{Rep}_{\text {cell }}^{G}\left(\mathcal{I}^{(n-1)}\right)$ is onto when $n \geq 2$. Let $\beta: \mathcal{I}^{(n-1)} \rightarrow G$ be a cellular representation. We must extend $\beta$ to $\hat{\beta}=\beta \cup\left\{\beta_{e}\right\} \in \operatorname{Rep}^{G}(\mathcal{I})$, which may be done for each $n$-cell independently. For each $e \in \Lambda_{n}(A)$, choose $f \in \Lambda_{n-1}(A)$ with $f \leq e$ and define $\beta_{e}=\beta_{f} \mid \mathcal{I}(e)$. We must check that $\beta_{e}$ does not depend on the choice of $f$. Let $f^{\prime}$ be another choice. As $n \geq 2$, there exists a continuous path $c(t)$ in the frontier of $|e|$ joining $a \in|f|$ to $a \in\left|f^{\prime}\right|$. By the face compatibility condition, $\beta_{a} \mid \mathcal{I}_{c(t)}$ is constant, thus $\beta_{f}\left|\mathcal{I}(e)=\beta_{f^{\prime}}\right| \mathcal{I}(e)$.

Corollary 5.2. Suppose that the hypotheses of Proposition 5.1 hold true. Let $b \in$ $\overline{\operatorname{Rep}}_{\text {cell }}^{G}(\mathcal{I})$. If $\operatorname{res}_{1}(b) \in \kappa\left(\operatorname{Rep}_{\text {cell }}^{G}\left(\mathcal{I}^{(1)}\right)\right)$, then $b \in \kappa\left(\operatorname{Rep}_{\text {cell }}^{G}(\mathcal{I})\right)$.

Proof. This is a consequence of the commutative diagram

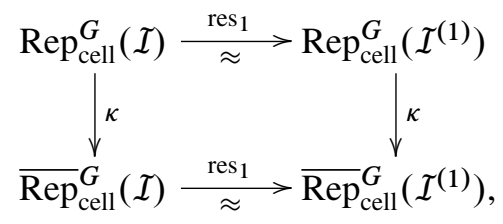

the bijectivity of the horizontal arrows coming from Proposition 5.1. 


\section{$\S 5$ B. Case where $G$ is abelian}

Proposition 5.3. Let $\mathcal{I}$ be a proper $(\Gamma, A)$-groupoid for a topological group $\Gamma$. Let $G$ be a compact abelian Lie group. Then the three maps $\imath, J, \kappa$ of diagram (10) are bijective.

Proof. The map $\kappa$ is bijective since conjugation has no effect if $G$ is abelian. It is then enough to prove that $\mathrm{J}$ is surjective. Let $\beta \in \operatorname{Rep}^{G}(\mathcal{I})$. As in the construction of $l$, one shows that $\beta(\zeta)=\beta\left(\zeta^{\prime}\right)$ if $e\left(\pi_{2}(\zeta)\right)=e\left(\pi_{2}\left(\zeta^{\prime}\right)\right)$, which is equivalent to $\beta$ being in the image of $J$.

If $\Gamma$ is a Lie group, Proposition 5.3 together with the classification Theorem 4.5 gives a bijection $\operatorname{SBun}_{\Gamma}^{G}(X) \approx \operatorname{Rep}_{\text {cell }}^{G}(\mathcal{I})$. Using Proposition 5.1 and the functorial property in diagram (5) (which holds true in the framework of Theorem 4.5), this

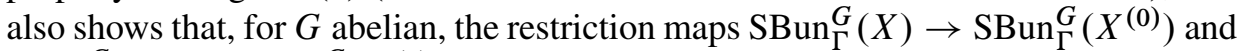
$\operatorname{SBun}_{\Gamma}^{G}(X) \rightarrow \operatorname{SBun}_{\Gamma}^{G}\left(X^{(1)}\right)$ are respectively injective and bijective, when $A$ is a regular $\mathrm{CW}$-complex.

By Lemma 1.3 , one has $G \stackrel{\approx}{\longrightarrow} G_{0} \times \pi_{0}(G)$, where $G_{0}$ is the identity component of the unit element. Therefore, $\operatorname{Rep}_{\text {cell }}^{G}(\mathcal{I}) \approx \operatorname{Rep}_{\text {cell }}^{\pi_{0}(G)}(\mathcal{I}) \times \operatorname{Rep}_{\text {cell }}^{G_{0}}(\mathcal{I})$ (the same decomposition holds for $\operatorname{SBun}_{\Gamma}^{G}(X)$ by equation (8), again true in the context of

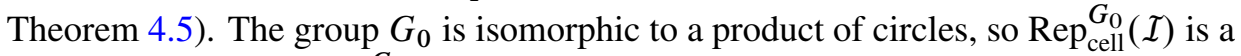
product of copies of $\operatorname{Rep}_{\text {cell }}^{G} s(\mathcal{I})$. We shall now study the latter.

§ 5C. $\operatorname{Rep}_{\text {cell }}^{G} \boldsymbol{s}(\mathcal{I})$ for $\mathcal{I}$ a toric groupoid. Let $\mathbb{T}$ be a torus. A cellular $(\mathbb{T}, A)$ groupoid $\mathcal{I}$ is called 0 -toric if $\mathcal{I}_{v}=\mathbb{T}$ for all $v \in \Lambda_{0}=\Lambda_{0}(A)$. It is called 1-toric if it is 0 -toric and if, for each $e \in \Lambda_{1}=\Lambda_{1}(A), \mathcal{I}(e)$ is a codimension 1 subtorus of $\mathbb{T}$. There is then $\chi_{e} \in \operatorname{Hom}\left(\mathbb{T}, S^{1}\right)$ with ker $\chi_{e}=\mathcal{I}(e)$. The part of $X$ above the closure $\|e\|$ of $|e|$ is a $\mathbb{T}$-space isomorphic to $\mathbb{C} P^{1}\left(\chi_{e}\right)$ of Example 4.14. The split $\mathbb{T}$-space with isotropy groupoid $\mathcal{I}^{(1)}$ is then a graph of $\mathbb{C} P^{1}(\chi)$ 's. Such a space $\mathbb{T}$ space $X$ is also called a GKM-space, as this property was first studied by M. Goresky, R. Kottwitz and R. MacPherson in [10].

Let $\mathrm{t}$ be the Lie algebra of $\mathbb{T}$ and let $\mathfrak{l}=\operatorname{ker}(\exp : \mathfrak{t} \rightarrow \mathbb{T})$. Moreover, let $\mathfrak{l}^{*}=$ $\left\{w \in \mathfrak{t}^{*} \mid w(\mathfrak{l}) \subset \mathbb{Z}\right\}$ (the dual lattice). Consider $S^{1}$ as $\mathbb{R} / \mathbb{Z}$. The correspondence which assigns to $\alpha \in \operatorname{Hom}\left(\mathbb{T}, S^{1}\right.$ ) its differential at the unit element of $\mathbb{T}$ (the weight of $\alpha$ ) produces an isomorphism between $\operatorname{Hom}\left(\mathbb{T}, S^{1}\right)$ and the additive group $\mathfrak{I}^{*}$. We shall thus identify $\operatorname{Hom}\left(\mathbb{T}, S^{1}\right)$ with $\mathfrak{l}^{*}$.

Let $\mathcal{I}$ be a 1-toric cellular $(\mathbb{T}, A)$-groupoid with $A$ a regular complex. By Proposition 5.1, $\operatorname{Rep}_{\text {cell }}^{G} s(\mathcal{I})$ injects into $\operatorname{Rep}_{\text {cell }}^{G} s\left(\mathcal{I}^{(0)}\right)$, which is the direct product of character groups

$$
\operatorname{Rep}_{\text {cell }}^{G} s(\mathcal{I}) \subset \prod_{v \in \Lambda_{0}} \operatorname{Hom}\left(\mathbb{T}, S^{1}\right)=\prod_{v \in \Lambda_{0}} \mathfrak{I}^{*}
$$

Let us orient each edge $e$; this determines an ordering $\partial_{-} e, \partial_{+} e$ of the two vertices of $e$. The character $\chi_{e}$ will also be seen in $\mathfrak{I}^{*}$. A family $\left(a_{v}\right)_{v \in \Lambda_{0}}$ is said to satisfy 
the GKM-conditions if, for each $e \in \Lambda_{1}$, the difference $a_{\partial_{+} e}-a_{\partial_{-} e}$ is a multiple of $\chi_{e}$. These conditions, considered in [10], are also discussed in Proposition 6.9 and Remark 6.10.

Proposition 5.4. Let $\mathcal{I}$ be a 1-toric cellular $(\mathbb{T}, A)$-groupoid. Then the image of $\operatorname{Rep}_{\text {cell }}^{G} S(\mathcal{I})$ in $\prod_{v \in \Lambda_{0}} \mathfrak{l}^{*}$ is the set offamilies $\left(a_{v}\right)_{v \in \Lambda_{0}}$ satisfying the GKM-condition.

Proof. By Proposition 5.1, it is enough to show that this condition characterises the image of $\operatorname{Rep}^{G}\left(\mathcal{I}^{(1)}\right)$ in $\operatorname{Rep}^{G}\left(\mathcal{I}^{(0)}\right)$. Denote by $\alpha_{v} \in \operatorname{Hom}\left(\mathbb{T}, S^{1}\right)$ the element with weight $a_{v} \in \mathfrak{I}^{*}$. For $e \in \Lambda_{1}$ the three following conditions are equivalent:

(a) the difference $a_{\partial_{+} e}-a_{\partial_{-} e}$ is a multiple of $\chi_{e}$.

(b) $\mathcal{I}(e) \subset \operatorname{ker} \alpha_{\partial_{+} e} \alpha_{\partial_{-} e}^{-1}$.

(c) $\alpha_{\partial_{+} e}\left|\mathcal{I}(e)=\alpha_{\partial_{-} e}\right| \mathcal{I}(e)$.

The equivalence between (a) and (b) comes from $\mathcal{I}(e)$ being of codimension 1 in $\mathbb{T}$. This proves Proposition 5.4.

Example 5.5. Let $X$ be a symplectic toric manifold of dimension $2 n$. It is a split $\mathbb{T}^{n}$-space, with $\pi: X \rightarrow A \subset \mathrm{t}^{*}$ being the moment map, and the isotropy groupoid $\mathcal{I}$ is 1 -toric. The moment polytope $A$ is a $n$ dimensional convex polytope of $\mathrm{t}^{*}$. It is known that each edge $e$ of $A$ is parallel to $\chi_{e}$ (see, e.g., [3], §4.2.4). By Proposition 5.4, $\operatorname{Rep}_{\text {cell }}^{G} s(\mathcal{I})$ may be visualised as the set of affine maps $\alpha: A \rightarrow \mathrm{t}^{*}$ such that $\alpha\left(\lambda_{0}(A)\right) \subset \mathfrak{I}^{*}$ and $\alpha(|e|)$ parallel to $\chi_{e}$ for each $e \in \lambda_{1}(A)$.

The left figure below shows a 2-dimensional moment polytope for a toric manifold, a Hirzebruch surface diffeomorphic to $\mathbb{C} P^{2} \sharp \overline{\mathbb{C} P^{2}}$. The torus $\mathbb{T}$ is $S^{1} \times S^{1}, \mathcal{I}_{12}=$ $I_{34}=\{1\} \times S^{1}, I_{14}=S^{1} \times\{1\}, I_{23}$ is the diagonal subgroup and the isotropy group for the 2-cell is trivial. The right figure visualises two elements of $\operatorname{Rep}^{G}(\mathcal{I})$.
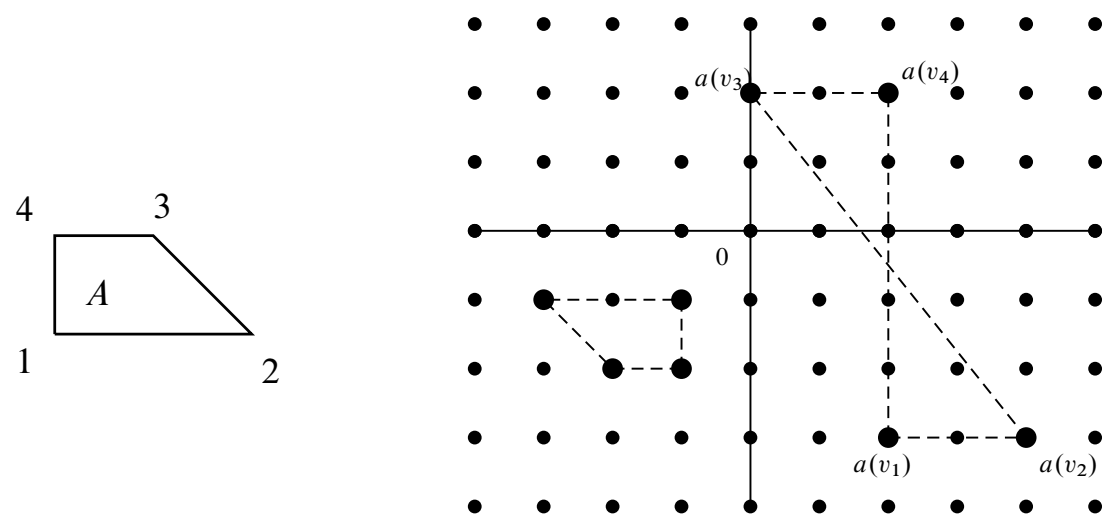

Let $\alpha \in \operatorname{Rep}_{\text {cell }}^{G} s(\mathcal{I})$. Let $X$ be the split $\mathbb{T}$-space with isotropy groupoid $\mathcal{I}$. Let $\eta$ be a split $\mathbb{T}$-equivariant $S^{1}$-principal bundle over $X$, with isotropy representation $\alpha$. 
Let $e \in \Lambda_{1}$. In Proposition 5.4, the integer $k_{e} \in \mathbb{Z}$ such that $a_{\partial_{+} e}-a_{\partial_{-}}=k_{e} \chi_{e}$ is related to the Euler number of $\eta$ restricted to $X_{e}$, the part of $X$ above the closure $\|e\|$ of $|e|$, which is homeomorphic to $\mathbb{C} P^{1}$. Choose a generator $\left[X_{e}\right]$ of $H_{2}\left(X_{e} ; \mathbb{Z}\right)$. Let $\varepsilon \in H^{2}\left(X_{e} ; \mathbb{Z}\right)$ be the Euler class of $\eta$ restricted to $X_{e}$.

Proposition 5.6. $\varepsilon\left(\left[X_{e}\right]\right)= \pm k_{e}$.

Proof. It is enough to consider the case where $X=X_{e}=\mathbb{C} P^{1}(\chi)$ for $\chi \in$ $\operatorname{Hom}\left(\mathbb{T}, S^{1}\right)$. The quotient space $A$ is then a segment, with two 0 -cells 0 and 1 and a 1-cell $e$, and we identify $A$ with $[0,1]$. One has $\mathcal{I}_{0}=\mathcal{I}_{1}=\mathbb{T}$ and $\mathcal{I}(e)=\operatorname{ker} \chi$. The elements $\alpha_{0}, \alpha_{1} \in \operatorname{Hom}\left(\mathbb{T} ; S^{1}\right)$ have weights $a_{0}, a_{1} \in \mathfrak{I}^{*}$. The bundle $\eta$ may then be identified with the bundle $E_{\alpha} \stackrel{\pi}{\rightarrow} Y_{\mathcal{I}}$ of the proof of Theorem 3.2.

Let $U_{0}=A-\{1\}$ and $U_{1}=A-\{0\}$ and call $\mathcal{W}_{0}$ and $\mathcal{W}_{1}$ the open sets of $X$ above $U_{0}$ and $U_{1}$. One has local sections $\sigma_{i}: \mathcal{W}_{i} \rightarrow E_{\alpha}$ of $\pi$ defined by $\sigma_{i}([\gamma, u])=$ $\left[\gamma, u, \alpha_{i}(\gamma)\right]$. Let $s \in \operatorname{Hom}\left(S^{1}, \mathbb{T}\right)$ such that $\chi \circ s: S^{1} \rightarrow \mathbb{T} / \mathcal{I}_{e}$ is surjective. Define $\hat{s}: S^{1} \rightarrow Y_{\mathcal{I}}$ by $\hat{s}(\delta)=[s(\delta), 1 / 2]$. One has

$$
\sigma_{1}(\hat{s}(\delta))=\sigma_{0}(\hat{s}(\delta)) \alpha_{0}(s(\delta))^{-1} \alpha_{1}(s(\delta))=\sigma_{0}(\hat{s}(\delta)) \cdot \chi(s(\delta))^{ \pm k_{e}}
$$

By the classification of $S^{1}$-principal bundles over a 2 -sphere, this proves Proposition 5.6.

§ 5D. Smooth circle bundles. Let $\mathcal{I}$ be a cellular $(\mathbb{T}, A)$-groupoid with $A$ a regular complex. Let $(X, \pi, \varphi)$ be a split $\mathbb{T}$-space with isotropy groupoid $\mathcal{I}$. Suppose that $X$ is (closed) smooth manifold and that the $\mathbb{T}$-action is smooth. In this subsection, we relate the isotropy representation $\Phi: \operatorname{SBun}_{\Gamma}^{G}(X) \rightarrow \operatorname{Rep}^{G}(\mathcal{I}) \approx \prod_{v \in \Lambda_{0}} \mathfrak{l}^{*}$ with some "moment map" $\Phi: X \rightarrow \mathrm{t}^{*}$. The material of this section is inspired by [14].

Let $\eta=(E \stackrel{p}{\rightarrow} X)$ be a smooth $\mathbb{T}$-equivariant split principal $S^{1}$-bundle over $X$. Choose $\theta \in \Omega^{1}(E)$ be an $\mathbb{T}$-invariant connection of the bundle $\eta$ (we see $S^{1}=\mathbb{R} / \mathbb{Z}$, so $\left.\operatorname{Lie}\left(S^{1}\right)=\mathbb{R}\right)$. This gives rise to a "moment map" $\Phi: X \rightarrow \mathrm{t}^{*}$ determined as follows. For $\xi \in \mathrm{t}$, denote by $\xi_{E}$ the vector field on $E$ induced by the action of $\mathbb{T}$. The map $\Phi$ is defined by the equation

$$
\langle\Phi(x), \xi\rangle=\theta\left(\xi_{E}(y)\right),
$$

for any $x \in X$ and $z \in p^{-1}(x)$. As $\theta$ is $\mathbb{T}$-invariant, the map $\Phi$ descends to a continuous map $\bar{\Phi}: A \rightarrow \mathrm{t}^{*}$.

Let $\alpha \in \operatorname{Rep}^{S^{1}}(\mathcal{I})$ be the isotropy representation of $\eta$. For each $v \in \Lambda_{0}(A)$, the homomorphism $\alpha_{v} \in \operatorname{Hom}\left(\mathbb{T}, S^{1}\right)$ is determined by its weight $a_{v} \in \mathfrak{I}^{*}$.

Proposition 5.7. Suppose that $\mathcal{I}$ is 0-toric. Then, for each $v \in \Lambda_{0}(A)$, one has $\bar{\Phi}(v)=a_{v}$. 
Proof. Let $\xi \in \mathrm{t}$. Let $v \in \Lambda_{0}(A)$ and $z \in E$ with $p(z)=\varphi(v)$. As $\varphi(v)$ is a fixed point, the vector $\xi_{E}(z)$ is tangent to the $S^{1}$-orbit $z \cdot S^{1}$. If we identify the latter with $S^{1}$, then $\theta\left(\xi_{E}(z)\right)$ is the derivative of $\alpha_{v}$, that is, $a_{v}$.

Remark 5.8. The figure of Example 5.5 suggests a possible relationship with the "twisted polytopes" of [14] which remains to be investigated.

$\S 5$ E. Case where $\boldsymbol{A}$ is a graph. In this section we shall determine $\operatorname{Rep}^{G}(\mathcal{I})$ for a $(\Gamma, A)$-groupoid $\mathcal{I}$ when $A$ is a graph, generalising the case treated in [13] where $A$ is a segment. One may suppose that the graph $A$ is regular. Indeed, the subdivision of an edge $e$, by adding a vertex $\hat{e} \in|e|$ and setting $\mathcal{I}_{\hat{e}}=\mathcal{I}(e)$, changes neither $\operatorname{Rep}^{G}(\mathcal{I})$ nor $\overline{\operatorname{Rep}}_{\text {cell }}^{G}(\mathcal{I})$. Observe also that if $G$ is connected, any $G$-principal bundle over $A$ is trivial, so for a split $\Gamma$-space $X$ over $A$ one has $\operatorname{SBun}_{\Gamma}^{G}(X)=\operatorname{Bun}_{\Gamma}^{G}(X)$. We start with some preliminary material.

Lemma 5.9. Let $\Gamma$ and $G$ be topological groups. Let $\mathcal{I}$ be a $(\Gamma, A)$-groupoid, where $A$ is a tree. Then $\kappa: \operatorname{Rep}_{\text {cell }}^{G}(\mathcal{I}) \rightarrow \overline{\operatorname{Rep}}_{\text {cell }}^{G}(\mathcal{I})$ is surjective.

Proof. The lemma is true for $A=\emptyset$ since then both $\operatorname{Rep}_{\text {cell }}^{G}(\mathcal{I})$ and $\overline{\operatorname{Rep}}_{\text {cell }}^{G}(\mathcal{I})$ are empty. Otherwise, let $b \in \overline{\operatorname{Rep}}_{\text {cell }}^{G}(\mathcal{I})$ and let $v$ be a vertex of $A$. Chose $\beta_{v} \in \operatorname{Hom}\left(\mathcal{I}_{v}, G\right)$ representing $b_{v}$. For an edge $e$ between $v$ and $v^{\prime}$, define $\beta_{e} \in$ $\operatorname{Hom}(\mathcal{I}(e), G)$ by $\beta_{e}=\beta_{v} \mid \mathcal{I}(e)$. Since $b \in \overline{\operatorname{Rep}}_{\text {cell }}^{G}(\mathcal{I})$, one can choose $\beta_{v^{\prime}} \in$ $\operatorname{Hom}\left(\mathcal{I}_{v^{\prime}}, G\right)$ which represents $b_{v^{\prime}}$ such that $\beta_{v^{\prime}} \mid \mathcal{I}(e)=\beta_{e}$. This constructs a cellular representation $\beta^{1}$ over the tree $A(v, 1)$ of points of distance $\leq 1$ from $v$ (for the distance where each edge has length 1). The same methods will propagate $\beta^{1}$ to $\beta^{2}$, over $A(v, 2)$ and then to $A(v, n)$ for all $n$. This defines $\beta \in \operatorname{Rep}_{\text {cell }}^{G}(\mathcal{I})$ with $\kappa(\beta)=b$.

Lemma 5.10. Let $\mathcal{I}$ be a $(\Gamma, A)$-groupoid, where $\Gamma$ is a topological group and $A$ is a graph. Let $G$ be a path-connected topological group. Then $\imath: \operatorname{Rep}^{G}(\mathcal{I}) \rightarrow$ $\overline{\operatorname{Rep}}_{\text {cell }}^{G}(\mathcal{I})$ is surjective.

Proof. We may suppose that $A$ is connected: otherwise, both $\operatorname{Rep}^{G}(\mathcal{I})$ and $\overline{\operatorname{Rep}}_{\text {cell }}^{G}(\mathcal{I})$ simply decompose into disjoint unions over components of $A$. Let $A_{0}$ be a maximal tree of $A$ and let $\mathcal{I}_{0}$ be the restriction of $\mathcal{I}$ over $A_{0}$. Let $b \in \overline{\operatorname{Rep}}_{\text {cell }}^{G}(\mathcal{I})$. By Lemma 5.9, there exists a cellular representation $\beta: \mathcal{I}_{0} \rightarrow G$ such that $\kappa(\beta)=b_{\mid I_{0}}$. We want to extend $\beta$ to $\hat{\beta}: \mathcal{I} \rightarrow G$. This can be done by defining $\hat{\beta}$ over $\|e\|$ for each edge $e$ of $A \backslash A_{0}$. Let $v, v^{\prime} \in \Lambda_{0}\left(A_{0}\right)$ be the vertices of $e$. As $b \in \overline{\operatorname{Rep}}_{\text {cell }}^{G}(\mathcal{I})$, there is $g \in G$ with $g^{-1} \beta_{v}(\gamma) g=\beta_{v^{\prime}}(\gamma)$ for all $\gamma \in \mathfrak{I}(e)$. Since $G$ is path-connected, there exists a continuous map $a \mapsto g_{a}$, from $\|e\|$ to $G$ with $g_{v}=1$ and $g_{v^{\prime}}=g$. For $a \in\|e\|$, we then define $\hat{\beta}_{a}: \mathcal{I}(e) \rightarrow G$ by $\hat{\beta}_{a}(\gamma)=g(a)^{-1} \beta_{v}(\gamma) g(a)$.

We now introduce some material in order to describe the preimage $l^{-1}(\alpha)$ of $\alpha \in \overline{\operatorname{Rep}}_{\text {cell }}^{G}(\mathcal{I})$. Let $K$ be a topological group and let $\tilde{\alpha} \in \operatorname{Hom}(K, G)$. Define $\mathcal{C}(\tilde{\alpha})$ 
to be the centraliser of $\tilde{\alpha}(K)$ in $G$. Let $\tilde{\alpha}^{\prime} \in \operatorname{Hom}(K, G)$ be such that $[\tilde{\alpha}]=\left[\tilde{\alpha}^{\prime}\right]$ in $\overline{\operatorname{Hom}}(K, G)$. Choose $b \in G$ such that $\tilde{\alpha}^{\prime}(\gamma)=b \tilde{\alpha}(\gamma) b^{-1}$. Sending $z \in \mathcal{C}(\tilde{\alpha})$ to $b z b^{-1}$ produces a continuous isomorphism $r_{\tilde{\alpha}^{\prime}, \tilde{\alpha}}: \mathcal{\ell}(\tilde{\alpha}) \rightarrow \mathcal{C}\left(\tilde{\alpha}^{\prime}\right)$ which does not depend on the choice of $b$. Moreover, one has $r_{\tilde{\alpha}^{\prime \prime}, \tilde{\alpha}} \circ r_{\tilde{\alpha}^{\prime \prime}, \tilde{\alpha}^{\prime}}=r_{\tilde{\alpha}^{\prime \prime}, \tilde{\alpha}}$. Therefore, a topological group $\ell(\alpha)$ is defined for $\alpha \in \overline{\operatorname{Hom}}(K, G)$ : take the disjoint union of $\mathcal{C}(\tilde{\alpha})$ for all representatives $\tilde{\alpha}$ of $\alpha$ and identify $z \in \mathcal{C}(\tilde{\alpha})$ with $r_{\tilde{\alpha}^{\prime}, \tilde{\alpha}}(z) \in \mathcal{C}\left(\tilde{\alpha}^{\prime}\right)$. If $K^{\prime}$ is a subgroup of $K$, one checks that $\mathcal{C}(\alpha)$ is a subgroup of $\mathcal{C}\left(\alpha_{\mid K^{\prime}}\right)$

Let $\mathcal{I}$ be a $(\Gamma, A)$-groupoid and $\alpha \in \overline{\operatorname{Rep}}_{\text {cell }}^{G}(\mathcal{I})$. Let $\dot{A}$ be the first barycentric subdivision of $A$. We assume that $A$ is regular, so $\Lambda_{1}(\dot{A})$ is the set of pairs $(v, e) \in \Lambda_{0}(A) \times \Lambda_{1}(A)$ with $v<e$; the edge corresponding to $(v, e)$ joins $v$ to the barycentre $\hat{e}$ of $e$. Form the group $X(\alpha)=\prod_{(v, e) \in \Lambda_{1}(\dot{A})} \pi_{0}\left(\mathcal{C}\left(\alpha_{e}\right)\right)$. Let $J^{0}: \prod_{v \in \Lambda_{0}(A)} \pi_{0}\left(\mathcal{C}\left(\alpha_{v}\right)\right) \rightarrow X(\alpha)$ be the homomorphism sending $\left(x_{v}\right)$ to $\left(z_{(w, e)}\right)$ with $z_{(w, e)}=j_{w, e}\left(x_{w}\right)$, where $j_{w, e}: \pi_{0}\left(\mathscr{C}\left(\alpha_{w}\right)\right) \rightarrow \pi_{0}\left(\mathcal{C}\left(\alpha_{e}\right)\right)$ is the homomorphism induced by the inclusion. Consider also the homomorphism

$J^{1}: \prod_{e \in \Lambda_{1}(A)} \pi_{0}\left(\mathcal{C}\left(\alpha_{e}\right)\right) \rightarrow X(\alpha)$ sending $\left(y_{e}\right)$ to $\left(z_{(w, f)}\right)$, where $z_{(w, f)}=y_{f}$. Set $Y^{0}(\alpha)$ and $Y^{1}(\alpha)$ to be the images of $J^{0}$ and $J^{1}$ and consider the double coset family $Z(\alpha)=Y^{0}(\alpha) \backslash X(\alpha) / Y^{1}(\alpha)$.

Theorem 5.11. Let $\mathcal{I}$ be a proper $(\Gamma, A)$-groupoid, with $\Gamma$ a topological group and $A$ a graph. Let $G$ be a compact connected Lie group. Then $\imath: \operatorname{Rep}^{G}(\mathcal{I}) \rightarrow \overline{\operatorname{Rep}}_{\text {cell }}^{G}(\mathcal{I})$ is surjective and the preimage $l^{-1}(\alpha)$ of $\alpha \in \overline{\operatorname{Rep}}_{\text {cell }}^{G}(\mathcal{I})$ is in bijection with $Z(\alpha)$.

Before proving Theorem 5.11, we state some of its corollaries, in which we assume the hypotheses of Theorem 5.11 and mention only the additional hypotheses.

Corollary 5.12. Let $A$ be a finite graph. Then the preimages of $\imath: \operatorname{Rep}^{G}(\mathcal{I}) \rightarrow$ $\overline{\operatorname{Rep}}_{\text {cell }}^{G}(\mathcal{I})$ are finite.

Proof. As $\mathcal{C}\left(\alpha_{e}\right)$ is a closed subgroup in $G, \pi_{0}\left(\mathcal{C}\left(\alpha_{e}\right)\right)$ is finite for each edge $e$ of $A$. Therefore $Z(\alpha)$ is finite.

The next corollary corresponds to [13], Theorem B and 8.12.

Corollary 5.13. Suppose that $\mathcal{I}$ is a proper $\left(\Gamma, \Delta^{1}\right)$-groupoid. Then the preimage $l^{-1}(\alpha)$ of $\alpha \in \overline{\operatorname{Rep}}_{\text {cell }}^{G}(\mathcal{I})$ is in bijection with the set of double cosets $\pi_{0}\left(\mathscr{C}\left(\alpha_{0}\right)\right) \backslash \pi_{0}\left(\mathscr{C}\left(\alpha_{01}\right)\right) / \pi_{0}\left(\mathscr{C}\left(\alpha_{1}\right)\right)$.

Proof. The group $X(\alpha)$ is isomorphic to $\pi_{0}\left(C\left(\alpha_{01}\right)\right) \times \pi_{0}\left(\ell\left(\alpha_{01}\right)\right)$ with $Y^{1}(\alpha) \approx$ $\pi_{0}\left(\mathcal{C}\left(\alpha_{01}\right)\right)$ being the diagonal subgroup. The group $Y^{0}(\alpha)$ is $\pi_{0}\left(\mathcal{C}\left(\alpha_{0}\right)\right) \times \pi_{0}\left(\mathcal{C}\left(\alpha_{1}\right)\right)$. Therefore, the map $X(\alpha) \rightarrow \pi_{0}\left(\complement\left(\alpha_{01}\right)\right)$ given by $\left(z_{0}, z_{1}\right) \mapsto z_{0} z_{1}^{-1}$ descends to a bijection from $Z(\alpha)$ to $\pi_{0}\left(\mathcal{C}\left(\alpha_{0}\right)\right) \backslash \pi_{0}\left(\mathscr{C}\left(\alpha_{01}\right)\right) / \pi_{0}\left(\mathcal{C}\left(\alpha_{1}\right)\right)$ (see [13], Section 8). 
Corollary 5.14. Suppose that $\mathcal{I}(e)$ is a torus for all edges e of A. Then it follows that $\imath: \operatorname{Rep}^{G}(\mathcal{I}) \rightarrow \overline{\operatorname{Rep}}_{\text {cell }}^{G}(\mathcal{I})$ is a bijection.

Proof. Let $\alpha \in \overline{\operatorname{Rep}}_{\text {cell }}^{G}(\mathcal{I})$ and let $\tilde{\alpha}: \mathcal{I} \rightarrow G$ be a continuous representation with $\imath(\tilde{\alpha})=\alpha$ Our hypotheses imply that $\tilde{\alpha}_{a}\left(\mathcal{I}_{e}\right)$ is a torus for all $e \in \Lambda_{1}(A)$ and all $a \in|e|$. As $G$ is connected, the group $\mathcal{C}\left(\alpha_{e}\right)$ is then connected (see, e.g., [8], Theorem 3.3.1). Therefore, $Z(\alpha)$ reduces to a single element.

Proof of Theorem 5.11. The surjectivity of $l$ is established in Lemma 5.10. Let $\alpha \in \overline{\operatorname{Rep}}_{\text {cell }}^{G}(\mathcal{I})$. The strategy is to construct a transitive action of $X(\alpha)$ on $t^{-1}(\alpha)$ and study the stabilisers.

Let $\tilde{\alpha}^{0}: \mathcal{I}^{(0)} \rightarrow G$ be a representative of $\alpha^{(0)}$. Let $\widetilde{\operatorname{Rep}}^{G}\left(\mathcal{I}, \tilde{\alpha}^{0}\right)$ be the set of continuous representations from $\mathcal{I}$ to $G$ which restrict to $\tilde{\alpha}^{0}$ on $\alpha^{(0)}$. As $G$ is connected, any map from $A^{(0)}$ to $G$ extends to $A$, which implies that each class in $l^{-1}(\alpha)$ has a representative in $\widetilde{\operatorname{Rep}}^{G}\left(\mathcal{I}, \tilde{\alpha}^{0}\right)$. Also, if $\tilde{\alpha} \in \widetilde{\operatorname{Rep}}^{G}\left(\mathcal{I}, \tilde{\alpha}^{0}\right)$, then $\imath(\tilde{\alpha})=\alpha$ by Proposition 5.1. Thus, the map $\tilde{\alpha} \mapsto[\tilde{\alpha}] \in \operatorname{Rep}^{G}(\mathcal{I})$ produces a surjection $\widetilde{\operatorname{Rep}^{G}}\left(\mathcal{I}, \tilde{\alpha}^{0}\right) \rightarrow l^{-1}(\alpha)$.

Form the group $\tilde{X}\left(\tilde{\alpha}^{0}\right)=\prod_{(v, e) \in \Lambda_{1}(\dot{A})} \mathcal{C}\left(\tilde{\alpha}^{0}(\mathcal{I}(e))\right.$. Let $z=\left(z_{(v, e)}\right) \in \tilde{X}\left(\tilde{\alpha}^{0}\right)$ and $\tilde{\alpha} \in \widetilde{\operatorname{Rep}}^{G}\left(\mathcal{I}, \tilde{\alpha}^{0}\right)$. For each edge $e$ of $A$ with $\partial e=\left\{v, v^{\prime}\right\}$, choose, using that $G$ is connected, a continuous map $g_{e}:\|e\| \rightarrow G$ such that $g_{e}(v)=z_{(v, e)}$ and $g_{e}\left(v^{\prime}\right)=$ $z_{\left(v^{\prime}, e\right)}$. We call $\left\{g_{e}\right\}$ a connecting family for $z$. Define $z \cdot\left\{g_{e}\right\} \tilde{\alpha} \in \widetilde{\operatorname{Rep}}^{G}\left(\mathcal{I}, \tilde{\alpha}^{0}\right)$ by

$$
z \cdot\left\{g_{e}\right\} \tilde{\alpha}(\gamma)= \begin{cases}g_{e}(a) \tilde{\alpha}(\gamma) g_{e}(a)^{-1} & \text { if } a \in|e| \text { and } \gamma \in \mathcal{I}_{a}, \\ \tilde{\alpha}_{a}(\gamma) & \text { otherwise. }\end{cases}
$$

For two connecting families $\left\{g_{e}\right\}$ and $\left\{\bar{g}_{e}\right\}$ for $z$, we check that

$$
z \cdot\left\{g_{e}\right\} \tilde{\alpha}(\gamma)=h(a)\left(z \cdot\left\{\bar{g}_{e}\right\} \tilde{\alpha}(\gamma)\right) h(a)^{-1},
$$

where $h: A \rightarrow G$ is the (continuous) map defined by $h(a)=g_{e}(a) \bar{g}_{e}(a)^{-1}$ if $a \in\|e\|$. This thus defines $z \cdot \tilde{\alpha}$ in $l^{-1}(\alpha)$ which does not depend on the choice of the connecting family $\left\{g_{e}\right\}$.

Now suppose that $\tilde{\alpha}, \tilde{\alpha}^{\prime} \in \widetilde{\operatorname{Rep}}^{G}\left(\mathcal{I}, \tilde{\alpha}^{0}\right)$ represent the same element in $\operatorname{Rep}^{G}(\mathcal{I})$. This means that there is a map $h: A \rightarrow G$ such that $\tilde{\alpha}_{a}^{\prime}(\gamma)=h(a) \tilde{\alpha}_{a}(\gamma) h(a)^{-1}$. Observe then that $h(v) \in \mathcal{C}\left(\tilde{\alpha}^{0}\left(\mathcal{I}_{v}\right)\right)$ for all $v \in \Lambda_{0}(A)$ and hence

$$
h(a)\left(z \cdot\left\{g_{e}\right\} \tilde{\alpha}\right) h(a)^{-1}=z \cdot\left\{h(a) g_{e} h(a)^{-1}\right\} \tilde{\alpha}^{\prime} .
$$

We have thus defined an action of $\tilde{X}\left(\tilde{\alpha}^{0}\right)$ on $l^{-1}(\alpha)$. We now prove that this action is transitive. Let $\tilde{\alpha}, \tilde{\alpha}^{\prime} \in \widetilde{\operatorname{Rep}}^{G}\left(\mathcal{I}, \tilde{\alpha}^{0}\right)$. Orient each edge $e$ of $A$, getting then $\partial e=\left\{\partial_{-} e, \partial_{+} e\right\}$. By Lemma 1.2, there exist $s, s^{\prime}:\|e\| \rightarrow G$ such that $\tilde{\alpha}(\gamma)=$ $s(a)^{-1} \tilde{\alpha}_{\partial_{-} e}^{0}(\gamma) s(a)$ and $\tilde{\alpha}^{\prime}(\gamma)=s^{\prime}(a)^{-1} \tilde{\alpha}_{\partial_{-} e}^{0}(\gamma) s^{\prime}(a)$ for all $\gamma \in \mathcal{I}(e)$ and all $a \in$ $\|e\|$. This implies that $s(\partial e)$ and $s^{\prime}\left(\partial_{-} e\right)$ are contained in $\mathcal{C}(\tilde{\alpha}(\mathcal{I}(e)))$. Hence one 
has $\tilde{\alpha}^{\prime}=z_{\left\{g_{e}\right\}} \cdot \tilde{\alpha}$, where $z_{\left(\partial_{-} e, e\right)}=s^{\prime}\left(\partial_{-} e\right)^{-1} s\left(\partial_{-} e\right), z_{\left(\partial_{+} e, e\right)}=s^{\prime}\left(\partial_{+} e\right)^{-1} s\left(\partial_{+} e\right)$ and $g_{e}(a)=s^{\prime}(a)^{-1} s(a)$. Hence the action of $\tilde{X}\left(\tilde{\alpha}^{0}\right)$ on $l^{-1}(\alpha)$ is transitive.

If $z=\left(z_{(v, e)}\right)$ is in the unit component of $\tilde{X}\left(\tilde{\alpha}^{0}\right)$, then $z \cdot\left\{g_{e}\right\} \tilde{\alpha}=\tilde{\alpha}$, if the maps $g_{e}$ are chosen so that $g(\hat{e})=1$ and $g_{e}(\|(v, e)\|) \subset \ell\left(\tilde{\alpha}_{v}^{0}(\mathcal{I}(e))\right)$. This implies that the action of $\tilde{X}\left(\tilde{\alpha}^{0}\right)$ on $l^{-1}(\alpha)$ descends to an action of the group $\prod_{(v, e) \in \Lambda_{1}(\dot{A})} \pi_{0}\left(\mathcal{C}\left(\tilde{\alpha}_{v}^{0}(\mathcal{I}(e))\right)\right.$ which is isomorphic to $X(\alpha)$.

Let $f$ be an edge of $A$, with $\partial f=\left\{v, v^{\prime}\right\}$. The representation $\tilde{\alpha}^{0}: I^{(0)} \rightarrow G$ can be chosen such that the restrictions to $\mathcal{I}_{e}$ of $\tilde{\alpha}_{v}^{0}$ and $\tilde{\alpha}_{v^{\prime}}^{0}$ coincide. For each $\zeta \in \mathcal{C}\left(\tilde{\alpha}_{v}^{0}(\mathcal{I}(f))=\mathcal{C}\left(\tilde{\alpha}_{v^{\prime}}^{0}(\mathcal{I}(f))\right.\right.$ we can then consider the element $z(\zeta)$ of $\tilde{X}\left(\tilde{\alpha}^{0}\right)$ satisfying $z_{(v, f)}(\zeta)=z_{\left(v^{\prime}, f\right)}(\zeta)=\zeta$ and $z_{(w, e)}(\zeta)=1$ of $e \neq f$. Then $z(\zeta) \cdot\left\{g_{e}\right\}$ $\tilde{\alpha}=\tilde{\alpha}$ if the $g_{e}$ are constant maps. This may be done for each edge $f$ of $A$, showing that the group $Y^{1}(\alpha)$ acts trivially on $\beta$ for all $\beta \in l^{-1}(\alpha)$.

Let $y \in \prod_{v \in \Lambda_{0}(A)}\left(\mathcal{C}\left(\tilde{\alpha}_{v}^{0}\left(\mathcal{I}_{v}\right)\right)\right)$ and $z \in \tilde{X}\left(\tilde{\alpha}^{0}\right)$. Consider the element $y z \in$ $\tilde{X}\left(\tilde{\alpha}^{0}\right)$ defined by $(y z)_{(v, e)}=y_{v} z_{(v, e)}$. Choose a connecting family $g_{e}:\|e\| \rightarrow G$ for $z$. For each $(v, e) \in \Lambda_{1}(\cdot A)$, choose $h_{(v, e)}:\|(v, e)\| \rightarrow G$ such that $h_{(v, e)}(v)=$ $y_{v}$ and $h_{(v, e)}(\hat{e})=1$. This defines a continuous map $h: A \rightarrow G$, by $h(a)=h_{(v, e)}(a)$ if $a \in\|(v, e)\|$, which conjugates $(y z){ }_{\left\{h g_{e}\right\}} \tilde{\alpha}$ with $\left.z \cdot{ }^{\prime} g_{e}\right\} \tilde{\alpha}$. This shows that $u x \cdot \beta=x \cdot \beta$ in $l^{-1}(\alpha)$, for all $u \in Y^{0}(\alpha), x \in X(\alpha)$ and $\beta \in l^{-1}(\alpha)$.

Fix $\beta \in l^{-1}(\alpha)$, represented by $\tilde{\beta} \in \widetilde{\operatorname{Rep}^{G}}\left(\mathcal{I}, \tilde{\alpha}^{0}\right)$. Consider the map $\tilde{\Psi}: \tilde{X}\left(\tilde{\alpha}^{0}\right) \rightarrow$ ${ }^{-1}(\alpha)$ given by $\tilde{\Psi}(z)=[z \cdot \tilde{\beta}]$. By the above, we have shown that $\tilde{\psi}$ descends to a surjection $\Psi: Z(\alpha) \rightarrow l^{-1}(\alpha)$. It remains to show that $\Psi$ is injective. Let $z^{\prime} \in \tilde{X}\left(\tilde{\alpha}^{0}\right)$ with $\Psi\left(z^{\prime}\right)=\Psi(z)$. Choose connecting families $\left\{g_{e}\right\}$ and $\left\{g_{e}^{\prime}\right\}$ for $z$ and $z^{\prime}$. If $\Psi\left(z^{\prime}\right)=\Psi(z)$, there exists a map $h: A \rightarrow G$ with $\left(z^{\prime} \cdot\left\{g_{e}^{\prime}\right\}\right.$ $\tilde{\beta})(\gamma)=h(a)\left(z \cdot\left\{g_{e}\right\} \tilde{\beta}\right)(\gamma) h(a)^{-1}$. Observe that $h(v) \in \mathcal{C}\left(\tilde{\alpha}^{0}\left(\mathcal{I}_{v}\right)\right)$ and therefore $h^{(0)}: \mathcal{I}^{(o)} \rightarrow G$ defines an element $y \in \prod_{v \in \Lambda_{0}(A)}\left(\mathcal{C}\left(\tilde{\alpha}_{v}^{0}\left(\mathcal{I}_{v}\right)\right)\right)$ satisfying $\left((y z) \cdot\left\{h g_{e}\right\} \tilde{\beta}\right)(\gamma)=h(a)\left(z \cdot\left\{g_{e}\right\} \tilde{\beta}\right)(\gamma) h(a)^{-1}$. Let $\bar{z}=y z$ and $\bar{g}_{e}=h g_{e}$. One has $[\bar{z}]=u[z]$ in $X(\alpha)$ with $u \in Y^{0}(\alpha)$. Thus, $\bar{z}$ and $z$ represent the same class in $Y^{0}(\alpha) \backslash X(\alpha)$ and the equality $z^{\prime} \cdot\left\{g_{e}^{\prime}\right\} \tilde{\beta}=\bar{z} \cdot\left\{\bar{g}_{e}\right\} \tilde{\beta}$ holds in $\widetilde{\operatorname{Rep}}^{G}\left(\mathcal{I}, \tilde{\alpha}^{0}\right)$. Therefore, $\bar{g}_{e}(a)^{-1} g_{e}^{\prime}(a) \in \mathcal{C}\left(\beta_{a}(\mathcal{I}(e))\right.$ for all $a \in\|e\|$. This implies that $z^{\prime}$ and $\bar{z}$ represent the same class in $X(\alpha) / Y^{1}(\alpha)$. Finally, we have shown that $z$ and $z^{\prime}$ represent the same class in $Z(\alpha)$, proving the injectivity of $\Psi$.

We now give some examples of the use of Theorem 5.11.

5.15. Let $A$ be the 1 -simplex $\Delta^{1}$. Let $\Gamma=\operatorname{SO}(n)$, with $n=2 k+1 \geq 3$ and consider the $(\Gamma, A)$-groupoid $\mathcal{I}$ with $\mathcal{I}_{0}=\mathcal{I}_{1}=\Gamma$ and $\mathcal{I}_{01}=\mathrm{SO}(n-1)$ (the split $\Gamma$-space $X$ with isotropy groupoid $\mathcal{I}$ is $S^{n}$ with the $\mathrm{SO}(n)$-action fixing the north and the south pole). For $G=\operatorname{SO}(n), \overline{\operatorname{Rep}}_{\text {cell }}^{G}(\mathcal{I})$ contains two elements, the trivial representation and the representation $\alpha$ with $\alpha_{0}=\alpha_{1}=$ id. The preimage by $l$ of the trivial representation contains one element but $l^{-1}(\alpha)$ contains two elements. For details and developments, see [13], Example 7.5. 
5.16. If $\pi_{1}(G)=\{1\}$, Theorem 5.11 extends to a $(\Gamma, A)$-groupoid $\mathcal{I}$ where $A$ is of dimension 2, provided that $I(e)=\{1\}$ when $e \in \Lambda_{2}(A)$. Examples are given by toric manifolds of (real) dimension 4.

5.17. Let $\mathcal{I}$ be the $S^{1}$-structure on the 1 -simplex $\Delta^{1}$ with $\mathcal{I}_{0}=\mathcal{I}_{1}=S^{1}$ and $\mathcal{I}_{01}=\{1\}$. The split $S^{1}$-space with isotropy groupoid $\mathcal{I}$ is $S^{2}$ with $S^{1}$ acting by rotation around an axis. By Theorem 5.11, $\operatorname{SBun}_{S^{1}}^{G}\left(S^{2}\right) \approx \overline{\operatorname{Hom}}\left(\mathcal{I}_{0}, G\right) \times \overline{\operatorname{Hom}}\left(\mathcal{I}_{1}, G\right)$. Choosing a maximal torus $\mathcal{T}$ in $G$, this yields $\operatorname{SBun}_{S^{1}}^{G}\left(S^{2}\right) \approx \operatorname{Hom}\left(\mathcal{I}_{0}, \mathcal{T}\right) / \mathcal{W} \times$ $\operatorname{Hom}\left(\mathcal{I}_{1}, \mathcal{T}\right) / \mathcal{W}$ where $\mathcal{W}$ is the Weyl group for $\mathcal{T}$. If $G$ is of $\operatorname{rank} k$, then $\operatorname{Hom}\left(\mathcal{I}_{0}, \mathcal{T}\right)$ and $\operatorname{Hom}\left(\mathcal{I}_{1}, \mathcal{T}\right)$ are both in bijection with $\mathbb{Z}^{k}$.

Let us specialise to $G=\mathrm{SO}(m)$ for $m \geq 3$. A maximal torus $\mathcal{T}$ of $\mathrm{SO}(m)$ is formed by matrices containing 2-blocks concentrated around the diagonal, so isomorphic to $\operatorname{SO}(2)^{k}$, and where $k=[m / 2]$. The action of $\mathcal{W}$ on $\operatorname{Hom}\left(S^{1}, \mathcal{T}\right) \approx \mathbb{Z}^{k}$ can be deduced from [1], p. 114. When $m=2 k+1$, the action of $\mathcal{W}$ on $\mathbb{Z}^{k}$ is generated by the permutation of coordinates and sign changes in any of them. A fundamental domain $\mathscr{D} \subset \mathbb{Z}^{k}$ is then

$$
\mathscr{D}=\left\{\left(r_{1}, \ldots, r_{k}\right) \in \mathbb{Z}^{k} \mid 0 \leq r_{1} \leq \cdots \leq r_{k}\right\}
$$

and $\operatorname{SBun}_{S^{1}}^{\operatorname{SO}(2 k+1)}\left(S^{2}\right) \approx \mathscr{D} \times \mathscr{D}$. When $m=2 k$, the sign changes must be even in number. A fundamental domain $\mathscr{E} \subset \mathbb{Z}^{k}$ is then

$$
\mathcal{E}=\left\{\left(r_{1}, \ldots, r_{k}\right) \in \mathbb{Z}^{k}\left|0 \leq r_{1} \leq \cdots \leq r_{k-1} \leq\right| r_{k} \mid\right\}
$$

and $\operatorname{SBun}^{\operatorname{SO}(2 k)}\left(S^{2}\right)_{S^{1}} \approx \varepsilon \times \varepsilon$.

This example was treated in our paper [13], Example 7.3, but the determination of $\operatorname{SBun}_{S^{1}}^{\mathrm{SO}(m)}\left(S^{2}\right)$ is wrong there because, in the action of the Weyl group, the sign changes were forgotten. However, the computation in [13], Example 7.3, of the second Stiefel-Whitney number $w_{2}(\xi)$ for $\xi \in \operatorname{SBun}^{\mathrm{SO}(m)}\left(S^{2}\right)_{S^{1}}$, being $\bmod 2$, is correct.

Here is an interesting consequence of the proof of Theorem 5.11.

Proposition 5.18. Let I be a proper $(\Gamma, A)$-groupoid, with A a regular $C W$-complex and $\Gamma$ a topological group. Let $G$ be a compact connected Lie group. Then $J: \operatorname{Rep}_{\text {cell }}^{G}(\mathcal{I}) \rightarrow \operatorname{Rep}^{G}(\mathcal{I})$ is injective.

Proof. As in the proof of Lemma 5.10, one may assume that $A$ is connected. Let $A_{0}$ be a maximal tree of $A$ and let $\mathcal{I}_{0}$ be the restriction of $\mathcal{I}$ over $A_{0}$. As $A$ is connected, $A_{0}$ contains all the vertices of $A$ and then the restriction map $\operatorname{Rep}_{\text {cell }}^{G}(\mathcal{I}) \rightarrow \operatorname{Rep}_{\text {cell }}^{G}\left(\mathcal{I}_{0}\right)$ is injective by Proposition 5.1. Therefore, it is enough to prove Proposition 5.18 when $A$ is a tree.

Let $\beta, \beta^{\prime}: \mathcal{I} \rightarrow G$ be cellular representations with $J(\beta)=J\left(\beta^{\prime}\right)$. Let $v$ be a vertex of the tree $A$. By conjugation of $\beta$ with a constant element of $G$, one may 
assume that $\beta_{v}=\beta_{v}^{\prime}$. Let $e$ be an edge between $v$ and $v^{\prime}$; one has $\beta_{e}=\beta_{e}^{\prime}$. Suppose that $\beta_{v^{\prime}} \neq \beta_{v^{\prime}}^{\prime}$. Then $\beta_{v}^{\prime}(\gamma)=z \beta(\gamma) z^{-1}$ with $z \in \mathcal{C}\left(\beta_{e}(\mathcal{I}(e))\right.$ and $z \notin \mathcal{C}\left(\beta_{v^{\prime}}\left(\mathcal{I}_{v^{\prime}}\right)\right)$. Choose a continuous map $g_{e}:\|e\| \rightarrow G$ with $g_{e}(v)=1$ and $g_{e}\left(v^{\prime}\right)=z$. Let $\mathcal{I}_{\|e\|}$ be the restriction of $\mathcal{I}$ over $\|e\|$ and let $\beta^{\prime \prime}: \mathcal{I}_{\|e\|} \rightarrow G$ be the (non-cellular) representation defined by $\beta^{\prime \prime}(\gamma)=g_{e}(a)^{-1} \beta^{\prime}(\gamma) g_{e}(a)$. Using the notations of the proof of Theorem 5.11, this means that $\beta, \beta^{\prime \prime} \in \widetilde{\operatorname{Rep}}^{G}\left(\mathcal{I}_{\|e\|}, \beta^{(0)}\right)$ and $\beta^{\prime \prime}=y \cdot g_{e} \beta$, where $y \in \tilde{X}\left(\beta^{(0)}\right)$ is defined by $y_{(v, e)}=1$ and $y_{\left(v^{\prime}, e\right)}=z$. The element $y$ is non-trivial in $Z\left(\alpha_{\|e\|}\right)$ which, by Theorem 5.11, would contradict the assumption $J(\beta)=J\left(\beta^{\prime}\right)$. Therefore, $\beta_{v^{\prime}}=\beta_{v^{\prime}}^{\prime}$. This argument may be done independently for all edges adjacent to $v$ and then propagated to the whole tree $A$.

When $A$ is a tree, the map $J: \operatorname{Rep}_{\text {cell }}^{G}(\mathcal{I}) \rightarrow \operatorname{Rep}^{G}(\mathcal{I})$ is actually bijective. More precisely:

Lemma 5.19. Let $\mathcal{I}$ be a proper $(\Gamma, A)$-groupoid, where $A$ is a graph and $\Gamma$ a topological group. Let $A_{0}$ be a subtree of $A$. Let $G$ be a compact Lie group. Then any $\alpha \in \operatorname{Rep}^{G}(\mathcal{I})$ has a representative which is cellular over $A_{0}$.

Proof. Let $v$ be a vertex of $A_{0}$. For an edge $e$ of $A_{0}$, between $v$ and $v^{\prime}$, there exists, by Lemma 1.2, a map $\psi_{e}:\|e\| \rightarrow G$ such that $\psi_{e}(a) \alpha_{a}(\gamma) \psi_{e}(a)^{-1}=\alpha_{v}(\gamma)$ for each $a \in\|e\|$ and $\gamma \in \mathcal{I}_{e}$. This defines a map $\psi_{1}: A_{0}(v, 1) \rightarrow G$ (notations as in the proof of Lemma 5.9). As $A_{0}(v, 1)$ is contractible, the homotopy extension property permits us to extend $\psi_{1}$ to a continuous map $\psi_{1}: A \rightarrow G$. The maps $\psi_{1}$ conjugates $\alpha$ to $\alpha_{1}$ which is cellular over $A_{0}(v, 1)$. The process propagates over $A_{0}(v, n)$ for all $n$, giving rise to a map $\psi: A \rightarrow G$ which conjugates $\alpha$ to a representation which is cellular over $A_{0}$.

Proposition 5.18 together with Lemma 5.19 implies the following

Corollary 5.20. Let $\mathcal{I}$ be a proper $(\Gamma, A)$-groupoid with $\Gamma$ a topological group and A a tree. Let $G$ be a compact connected Lie group. Then $J: \operatorname{Rep}_{\text {cell }}^{G}(\mathcal{I}) \rightarrow \operatorname{Rep}^{G}(\mathcal{I})$ is bijective.

5.21. In contrast with Theorem 5.11 , the map $J: \operatorname{Rep}_{\text {cell }}^{G}(\mathcal{I}) \rightarrow \operatorname{Rep}^{G}(\mathcal{I})$ is not surjective when the graph $A$ is not a tree. Using Lemma 5.9, it is enough to find an example where $\kappa: \operatorname{Rep}_{\text {cell }}^{G}(\mathcal{I}) \rightarrow \overline{\operatorname{Rep}}_{\text {cell }}^{G}(\mathcal{I})$ is not surjective. Let $A$ be the 1skeleton of the 2-simplex $\Delta^{2}$ with $\mathcal{I}_{0}=\mathcal{I}_{1}=I_{2}=\Gamma=S^{1} \times S^{1}, I_{01}=1 \times S^{1}$, $\mathcal{I}_{02}=S^{1} \times 1$ and $\mathcal{I}_{12}$ is the diagonal $S^{1}$. The split $\Gamma$-space with this isotropy groupoid is $\mathbb{C} P^{2}$ with the action $\left.\left.\left(c_{1}, c_{2}\right) \cdot\left[z_{0}: z_{1}: z_{2}\right)\right]=\left[c_{0} z_{0}: c_{1} z_{1}: z_{2}\right)\right]$. Take $G=\mathrm{SU}(2)$; the diagonal torus $H$ has dimension 1 and its Weyl group $\mathcal{W}$ acts by passing to the inverse. Then

$$
\begin{aligned}
\overline{\operatorname{Hom}}(\Gamma, \operatorname{SU}(2)) & \approx \operatorname{Hom}(\Gamma, H) / \mathcal{W} \\
& \approx \hat{\Gamma} /\{\chi \sim-\chi\} \approx(\mathbb{Z} \times \mathbb{Z}) /\{(p, q) \sim-(p, q)\} .
\end{aligned}
$$


We identify $\overline{\operatorname{Hom}}(\Gamma, \mathrm{SU}(2))$ with the fundamental domain $\mathscr{D}$ in $\mathbb{Z} \times \mathbb{Z}$ :

$$
\mathscr{D}:=\{(p, q) \in \mathbb{Z} \times \mathbb{Z}) \mid q \geq 0 \text { and }(p \geq 0 \text { if } q=0)\} .
$$

If $\beta \in \operatorname{Rep}_{\text {cell }}^{G}(\mathcal{I})$ is not trivial, it must be not trivial on at least one edge-isotropy groups (say $\mathcal{I}_{01}$ ). Then $\beta$ is conjugate to $\beta^{\prime}$ such that $\beta_{01}^{\prime}\left(\mathcal{I}_{01}\right) \subset H$. As $H$ is maximal abelian, $\beta^{\prime}$ is then an algebraic representation of $\mathcal{I}$ in $H$. By Proposition 5.4, one has an identification of $\operatorname{Rep}^{H}(\mathcal{I})$ with the set of triples

$$
\left(\left(p_{0}, q_{0}\right),\left(p_{1}, q_{1}\right),\left(p_{2}, q_{2}\right)\right) \in(\mathbb{Z} \times \mathbb{Z})^{3}
$$

such that

A class in $\overline{\operatorname{Rep}}_{\text {cell }}^{G}(\mathcal{I})$ is a triple

$$
p_{0}=p_{2}, \quad q_{0}=q_{1}, \quad \text { and } \quad p_{1}+q_{1}=p_{2}+q_{2} .
$$

$$
\left(\left[p_{0}, q_{0}\right],\left[p_{1}, q_{1}\right],\left[p_{2}, q_{2}\right]\right) \in \mathscr{D} \times \mathscr{D} \times \mathscr{D}
$$

such that $\left|p_{0}\right|=\left|p_{2}\right|, q_{0}=q_{1}$ and $\left|p_{1}+q_{1}\right|=\left|p_{2}+q_{2}\right|$. The class $\alpha \in \overline{\operatorname{Rep}}_{\text {cell }}^{G}(\mathcal{I})$ corresponding to $([-1,2],[3,2],[1,4])$ is not in the image of $\kappa$. Indeed, none of the 8 triples in $(\mathbb{Z} \times \mathbb{Z})^{3}$ above $\alpha$ satisfies equations (14).

\section{Comparison with the homotopy-theoretic approach}

$\S 6$ A. Haefliger classifying spaces. Let $(X, \pi, \varphi)$ be a split $\Gamma$-space over a space $A$ with isotropy groupoid $\mathcal{I}$. Let $B \mathcal{I}$ be the Haefliger classifying space for $\mathcal{I}$ [12], p. 140. For a groupoid like $B \mathcal{I}$ where morphisms go from one object to itself, we check that the construction of [12], p. 140, takes the following form: set

$$
E \mathcal{I}=\left\{(v, a) \in E \Gamma \times A \mid v \in E \mathcal{I}_{a}\right\},
$$

with the induced topology, and define $B I$ as the quotient space $E \mathcal{I} / \mathcal{I}$. The projection $\bar{\pi}: B \mathcal{I} \rightarrow A$ makes $B \mathcal{I}$ is a space over $A$ whose stalk over $a$ is the Milnor classifying space $B \mathcal{I}_{a}$. There is a section $j: A \rightarrow B \mathcal{I}$ of $\bar{\pi}$ sending $a \in A$ to the class of $\left(v_{0}, a\right)$ where $v_{0}=(1 e, 0, \ldots) \in E \Gamma$, expressed as the infinite join, with $e$ the unit element of $\Gamma$. The inclusion $\mathcal{I} \subset \Gamma \times A$ is a morphism of topological groupoids and therefore induces a continuous map $E \mathcal{I} \rightarrow E \Gamma \times A$ which descends to a continuous map $B \mathcal{I} \rightarrow B \Gamma \times A$.

Recall that the Borel construction associates to $X$ the space $X_{\Gamma}=E \Gamma \times_{\Gamma} X$. The map $\pi: X \rightarrow A$ descends to a continuous and open surjective map $\bar{\pi}: X_{\Gamma} \rightarrow A$, with $\bar{\pi}^{-1}(a)=E \Gamma \times_{\Gamma} \mathcal{I}_{a} \approx B \mathcal{I}_{a}$. The composed map $E \mathcal{I} \rightarrow E \Gamma \times A \stackrel{\operatorname{id} \times \varphi}{\longrightarrow} E \Gamma \times X$ descends to a continuous map $\delta: B \mathcal{I} \rightarrow X_{\Gamma}$ over the identity of $A$. The restriction of $\delta$ to each stalk is a weak homotopy equivalence. It would then be interesting to figure out, for instance in the spirit of Sections 2 and 3, under which hypotheses $\delta$ is a weak homotopy equivalence. We will restrict ourselves to $(\Gamma, A)$-groupoids, where we get the following proposition. 
Proposition 6.1. Let $\mathcal{I}$ be a proper $(\Gamma, A)$-groupoid for a Lie group $\Gamma$. Let $(X, \pi, \varphi)$ be a split $\Gamma$-space over $A$, with isotropy groupoid $\mathcal{I}$. Then the map $\delta: B \mathcal{I} \rightarrow X_{\Gamma}$ is a homotopy equivalence.

Proof. By Proposition 4.3, we may suppose that $(X, \pi, \varphi)=\left(Y_{\mathcal{I}}, \Pi, \phi\right)$. If $K$ is a subspace of $A$, we denote by $\mathcal{I}(K)$ the subgroupoid of $\mathcal{I}$ formed by all the stalks over $K$, and we set $X(K)=Y_{\mathcal{I}(K)}$.

Observe first that Proposition 6.1 is true if $\mathcal{I}_{a}$ is constant for all $a \in A$. Indeed, one then has $X=\Gamma / \mathcal{I}_{a} \times A$, so $B \mathcal{I} \approx B \mathcal{I}_{a} \times A$ and $X_{\Gamma} \approx E \Gamma / \mathcal{I}_{a} \times A$ and $\delta$ is a homotopy equivalence. More generally, Proposition 6.1 remains true if $\mathcal{I}_{a}$ is locally constant, meaning constant on each connected component of $A$.

Proposition 6.1 will be proved, by induction on $n$, for $X\left(A^{(n)}\right)$, the split $\Gamma$-space over the $n$-skeleton $A^{(n)}$ of $A$. It is true for $n=0$ since $\mathcal{I}\left(A^{(0)}\right)$ is locally constant. The induction step involves the subcomplexes $K^{\prime}=A^{(n-1)} \subset K=A^{(n)}$, so $K$ is obtained from $K^{\prime}$ by adjunction of $\mathcal{E}=\bigsqcup_{e \in \Lambda_{n}} D_{e}^{n}$, via the attaching map $f: \partial \mathcal{E}=\bigsqcup_{e \in \Lambda_{n}} S_{e}^{n-1} \rightarrow K^{\prime}\left(\Lambda_{n}=\Lambda_{n}(A)\right)$. Then $X(K)$ is obtained from $X\left(K^{\prime}\right)$ by attaching the $\Gamma$-space $\tilde{\mathcal{E}}=\coprod_{e \in \Lambda_{n}}\left(\Gamma / \mathcal{I}(e) \times D_{e}^{n}\right)$ via the $\Gamma$-equivariant map $\tilde{f}: \partial \tilde{\mathcal{E}}=\bigsqcup_{e \in \Lambda_{n}}\left(\Gamma / \mathcal{I}(e) \times S_{e}^{n-1}\right) \rightarrow X\left(K^{\prime}\right)$. We denote by $F: \mathcal{E} \rightarrow K$ and $\tilde{F}: \tilde{\mathcal{E}} \rightarrow X(K)$ the characteristic maps, extending $f$ and $\tilde{f}$. We see $\tilde{\mathcal{E}}$ and $\partial \tilde{\mathcal{E}}$ as split $\Gamma$-spaces over $\mathcal{E}$ and $\partial \mathcal{E}$ respectively with locally constant isotropy groupoids: if $x \in D_{e}^{n}$, then $\mathcal{I}(\mathscr{E})=\mathcal{I}(\partial \mathcal{E})=\mathcal{I}(e)$. Let us consider the following diagram:

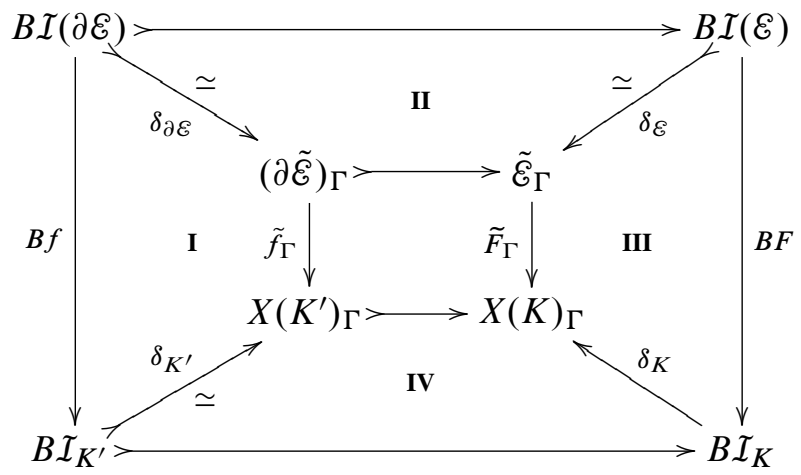

The maps $\delta_{\partial \mathcal{E}}$ and $\delta_{\mathcal{E}}$ are homotopy equivalences since the isotropy groupoids are locally constant. The map $\delta_{K^{\prime}}$ is a homotopy equivalence by induction hypothesis. Restriction to any stalk shows that diagrams I-IV are commutative. As $\Gamma$ is a Lie group, all the spaces under consideration have the homotopy type of $\mathrm{CW}$-complexes. Therefore, the outer and inner square diagrams are homotopy push-out diagrams. By push-out properties, the map $\delta_{K}$ is a homotopy equivalence.

§ 6B. Split bundles and classifying spaces. Let $\eta:(P \stackrel{p}{\rightarrow} X)$ be a $\Gamma$-equivariant principal $G$-bundle. The Borel construction $E \Gamma \times_{\Gamma} P \rightarrow E \Gamma \times_{\Gamma} X$ yields a 
principal $G$-bundle $\eta_{\Gamma}$ over $X_{\Gamma}$ with the same trivialising cover as $\eta$. If $\eta$ is numerable, so is $\eta_{\Gamma}$. Let $(X, \pi, \varphi)$ be a split $\Gamma$-CW-complex over $A$. By Theorem 4.5, any split $\Gamma$-equivariant principal bundle over $X$ is numerable. Therefore we obtain a map $\Psi: \operatorname{Bun}_{\Gamma}^{G}(X) \rightarrow\left[X_{\Gamma}, B G\right]$. The isotropy representation $\Phi: \operatorname{SBun}_{\Gamma}^{G}(X) \stackrel{\approx}{\approx} \operatorname{Rep}^{G}(\mathcal{I})$ is a bijection. Passing to the classifying spaces gives a map $B: \operatorname{Rep}^{G}(\mathcal{I}) \rightarrow[B \mathcal{I}, B G]$. The map $\delta: B \mathcal{I} \rightarrow X_{\Gamma}$ of Section $\S 6$ A gives rise to a map $\delta^{*}:\left[X_{\Gamma}, B G\right] \rightarrow[B \mathcal{I}, B G]$.

Proposition 6.2. Let I be a proper $(\Gamma, A)$-groupoid for a Lie group $\Gamma$. Let $(X, \pi, \varphi)$ be a split $\Gamma$-CW-complex over $A$ with isotropy groupoid $\mathcal{I}$. Let $G$ be a compact Lie group. Then the diagram

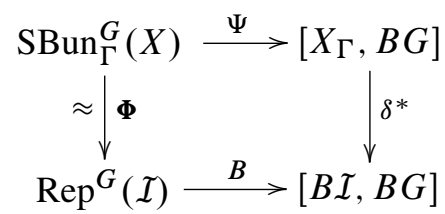

is commutative.

Proof. By Proposition 4.3, we may assume that $(X, \pi, \varphi)=\left(Y_{\mathfrak{I}}, \Pi, \phi\right)$. Let $\varepsilon \in$ $\operatorname{SBun}_{\Gamma}^{G}\left(Y_{\mathcal{I}}\right)$ and let $\epsilon: \mathcal{I} \rightarrow G$ be a representatative of $\boldsymbol{\Phi}(\varepsilon)$. By Theorem 4.5 and its proof, $\varepsilon$ has a representative $\eta$ of the form

$$
\Gamma \times_{\mathfrak{I}}(A \times G) \rightarrow \Gamma \times \times_{\mathfrak{I}} A=Y_{\mathfrak{I}},
$$

where $\mathcal{I}$ acts on $A \times G$ by $\zeta \cdot(a, g)=(a, \epsilon(\zeta) g)$. The bundle $\eta_{\Gamma}$ takes the form

$$
E \Gamma \times_{\mathcal{I}}(A \times G) \rightarrow E \Gamma \times{ }_{I} A=X_{\Gamma} .
$$

Let $q: L \rightarrow B \mathcal{I}$ be the induced bundle $\delta^{*} \eta_{\Gamma}$. To prove Proposition 6.2, it is enough to construct a $G$-equivariant map $F: L \rightarrow E G$ making the diagram

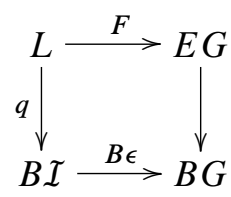

commutative. Restricted to the stalk over $a$, the bundle $\delta^{*} \eta_{\Gamma}$ is of the form

$$
E \mathcal{I}_{a} \times \mathcal{I}_{a}(\{a\} \times G) \longrightarrow E \mathcal{I}_{a} \times \mathcal{I}_{a}\{a\} .
$$

Therefore, the required map $F$ can be defined by

$$
F(u, a, g)=E \epsilon(u) \cdot g .
$$


Proposition 6.2 allows us to study the map $B: \operatorname{Rep}^{G}(\mathcal{I}) \rightarrow[B \mathcal{I}, B G]$, especially when $G$ is abelian, in which case $B$ is a homomorphism of abelian groups.

Proposition 6.3. Let $\mathcal{I}$ be a proper $(\Gamma, A)$-groupoid for a Lie group $\Gamma$. Let $(X, \pi, \varphi)$ be a split $\Gamma-C W$-complex over $A$ with isotropy groupoid $\mathcal{I}$. Let $G$ be a compact abelian Lie group. Then one has an isomorphism of split exact sequences of abelian groups:

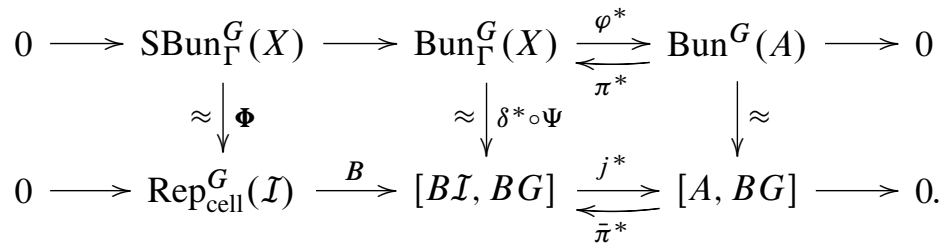

Proof. The top split exact sequence of abelian groups comes from Proposition 4.7 and its proof. For the bottom one, one has at least a sequence

$$
\operatorname{Rep}^{G}(\mathcal{I}) \stackrel{B}{\rightarrow}[B \mathcal{I}, B G] \stackrel{j^{*}}{\rightarrow}[A, B G]
$$

with $j^{*} \circ B=0$. By Proposition 4.7 , any principal $\Gamma$-equivariant $G$-bundle over $X$ is numerable. Therefore, the map $\delta^{*} \circ \Psi$ is defined and is a homomorphism of abelian groups. One checks that the left-hand square of the diagram (15) is commutative, as well as the right-hand square with $\varphi^{*}$ and $j^{*}$. The map $\delta^{*}$ is bijective by Proposition 6.1. As $G$ is abelian, the map $\Psi$ is a bijection by [18], Theorem A. Thus, $\delta^{*} \circ \Psi$ is an isomorphism. This proves that the bottom sequence of diagram (15) is split exact.

Corollary 6.4. Let I be a proper $(\Gamma, A)$-groupoidfor a Lie group $\Gamma$. Let $(X, \pi, \varphi)$ be a split $\Gamma$-space over $A$ with isotropy groupoid $\mathcal{I}$. Let $G$ be a compact abelian Lie group. Suppose that $H^{1}\left(A ; \pi_{0}(G)\right)=H^{2}(A ; \mathbb{Z})=0$. Then the map $B: \operatorname{Rep}^{G}(\mathcal{I}) \rightarrow$ $[B \mathcal{I}, B G]$ is a bijection.

Proof. The group $G$ is a disjoint union of tori, so $\pi_{j}(B G)=\pi_{j-1}(G)=0$ for $j>2$. One has $\operatorname{Hom}\left(\pi_{1}(A), \pi_{1}(B G)\right)=\operatorname{Hom}\left(\pi_{1}(A), \pi_{0}(G)\right) \approx H^{1}\left(A ; \pi_{0}(G)\right)=0$. A map $f: A \rightarrow B G$ is then null-homotopic on the 1-skeleton and the obstruction theory to homotop it to a constant map is with constant coefficients. Our hypotheses implies that $H^{2}\left(A ; \pi_{2}(B G)\right)=0$, so one gets $[A, B G]=0$. Corollary 6.4 then follows from Proposition 6.3.

6.5. Equivariant K-theory. For vector bundles it is natural to stabilize, and then to study bundles via equivariant K-theory. For example, if $G=U(n)$ we consider the stabilization maps

$$
\operatorname{SBun}_{\Gamma}^{U(n)}(X) \rightarrow \operatorname{SBun}_{\Gamma}^{U(n+1)}(X)
$$

and point out how stabilization is related to our classification results. 
Proposition 6.6. Let I be a proper $(\Gamma, A)$-groupoid for a Lie group $\Gamma$. Let $(X, \pi, \varphi)$ be a split $\Gamma$-CW-complex over $A$ with isotropy groupoid $\mathcal{I}$. Then there is a natural isomorphism

$$
\boldsymbol{\Phi}: K_{\Gamma}(X, A) \cong \operatorname{KRep}(\mathcal{I})
$$

of abelian groups induced by the isotropy representations.

The group $\operatorname{KRep}(\mathcal{I})$ is the Grothendieck group of the abelian monoid obtained by stabilization from the system $\left\{\operatorname{Rep}^{U(n)}(\mathcal{I})\right\}$.

6.7. Equivariant cohomology. Let $\Gamma$ be a compact Lie group and $X$ a $\Gamma$-CWcomplex. By [18], Theorem A, one has isomorphisms

$$
\operatorname{Bun}_{\Gamma}^{S^{1}}(X) \stackrel{\approx}{\longrightarrow}\left[X_{\Gamma}, B S^{1}\right] \approx H_{\Gamma}^{2}(X),
$$

where $H_{\Gamma}^{*}(X)=H_{\Gamma}^{*}(X ; \mathbb{Z})$ denotes the equivariant cohomology. If $(X, \pi, \varphi)$ is a split $\Gamma$-space over a CW-complex $A$, then the projection $\pi$ descends to a map $\bar{\pi}: X_{\Gamma} \rightarrow A$. We denote by $X^{(i)}$ the part of $X$ above the $i$-skeleton of $A$ and by $r_{i}: H_{\Gamma}^{*}(X) \rightarrow H_{\Gamma}^{*}\left(X^{(i)}\right)$ the restriction homomorphism induced by the inclusion $X^{(i)} \subset X$.

Proposition 6.8. Let $\Gamma$ be topological group and $A$ be a $C W$-complex. Let I be a proper $(\Gamma, A)$-groupoid and $(X, \pi, \varphi)$ be a split $\Gamma$-space with isotropy groupoid $\mathcal{I}$. Then

(a) the sequence $0 \rightarrow H^{2}(A) \stackrel{\bar{\pi}^{*}}{\longrightarrow} H_{\Gamma}^{2}(X) \stackrel{r_{0}}{\rightarrow} H_{\Gamma}^{2}\left(X^{(0)}\right)$ is exact;

(b) the two restriction homomorphisms

$$
r_{0}: H_{\Gamma}^{2}(X) \rightarrow H_{\Gamma}^{2}\left(X^{(0)}\right) \text { and } r_{10}: H_{\Gamma}^{2}\left(X^{(1)}\right) \rightarrow H_{\Gamma}^{2}\left(X^{(0)}\right)
$$

have the same image.

Proof. The map $\bar{\pi}$ admits a section $\bar{\varphi}: A \rightarrow X_{\Gamma}$ coming from $\varphi$. Therefore, $\bar{\pi}^{*}: H^{*}(A) \rightarrow H_{\Gamma}^{*}(X)$ is injective.

One has $H_{\Gamma}^{2}\left(X^{(0)}\right) \approx \operatorname{SBun}^{S^{1}} X^{(0)} \approx \operatorname{Rep}_{\text {cell }}^{G} s\left(\mathcal{I}^{(0)}\right)$. The composed homomorphism $\operatorname{Bun}^{S^{1}}(A) \approx H^{2}(A) \stackrel{\pi^{*}}{\longrightarrow} H_{\Gamma}^{2}(X) \stackrel{r_{0}}{\rightarrow} H_{\Gamma}^{2}\left(X^{(0)}\right) \approx \operatorname{Rep}_{\text {cell }}^{G} s\left(\mathcal{I}^{(0)}\right)$ sends an $S^{1}$-bundle $\xi$ over $A$ to the isotropy representation of $\pi^{*} \xi$, which is trivial. Thus, $r_{0} \circ \bar{\pi}^{*}=0$. Using Proposition 3.5, one has an isomorphism $H_{\Gamma}^{2}(X) \approx$ $\operatorname{Rep}_{\text {cell }}^{G} s(\mathcal{I}) \times H^{2}(A)$. The remainder of (a) and (b) follow from Proposition 5.1.

We now specialise to $\Gamma$ being a torus $\mathbb{T}$, with Lie algebra $\mathfrak{l}$, and use the definitions and notations of Section $\S 5$ C. If $\mathcal{I}$ is 0 -toric, we have from equation (12), that

$$
H_{\mathbb{T}}^{2}\left(X^{(0)}\right) \approx \operatorname{Rep}_{\text {cell }}^{G} s\left(\mathcal{I}^{(0)}\right) \approx \prod_{v \in \Lambda_{0}} \mathfrak{r}^{*}
$$

by using Proposition 6.8 and its proof, together with Proposition 5.4. 
Proposition 6.9. Let $\mathcal{I}$ be a 1 -toric cellular $(\mathbb{T}, A)$-groupoid and let $(X, \pi, \varphi)$ be a split $\mathbb{T}$-space with isotropy groupoid $\mathcal{I}$. The image of $r: H_{\mathbb{T}}^{2}(X) \rightarrow \prod_{v \in \Lambda_{0}} \mathfrak{I}^{*}$ is the set of elements $\left(a_{v}\right)_{v \in \Lambda_{0}}$ satisfying the GKM-condition.

Remark 6.10. Let $X$ be as in Proposition 6.9. Suppose that $X$ is equivariantly formal, i.e. the homomorphism $H_{\mathbb{T}}^{*}(X) \rightarrow H^{*}(X)$ induced by the inclusion $X \subset X_{\mathbb{T}}$ is surjective. In this case, the homomorphim $H_{\Gamma}^{2}(X) \stackrel{r_{0}}{\rightarrow} H_{\Gamma}^{2}\left(X^{(0)}\right)$ is injective and Part (b) of Proposition 6.8 holds, see [9], Theorem 1 . The injectivity of $r_{0}$ is considered as a "localisation theorem", see e.g., [10], Theorem 6.3, and Part (b) of Proposition 6.8 is referred to as the "Chang-Skjelbred principle" (historically it occurred in [5], Lemma 2.3, for rational coefficients). But, by Part (a) of Proposition 6.8, $X$ is equivariantly formal only if $H^{2}(A)=0$, so our context is different. For complex coefficients, Proposition 6.9 was proven in [10], Theorem 7.2. There $X$ need not to be split but again must be equivariantly formal.

\section{References}

[1] J. F. Adams, Lectures on Lie groups. W. A. Benjamin, Inc., New York 1969. Zbl 0206.31604 MR 0252560

[2] S. Adams, Reduction of cocycles with hyperbolic targets. Ergodic Theory Dynam. Systems 16 (1996), 1111-1145. Zbl 0869.58031 MR 1424391

[3] M. Audin, The topology of torus actions on symplectic manifolds. Progr. Math. 93, Birkhäuser Verlag, Basel 1991. Zbl 0726.57029 MR 1106194

[4] M. R. Bridson and A. Haefliger, Metric spaces of non-positive curvature. Grundlehren Math. Wiss. 319, Springer-Verlag, Berlin 1999. Zbl 0988.53001 MR 1744486

[5] T. Chang and T. Skjelbred, The topological Schur lemma and related results. Ann. of Math. (2) 100 (1974), 307-321. Zbl 0288.57021 MR 0375357

[6] M.W. Davis and T. Januszkiewicz, Convex polytopes, Coxeter orbifolds and torus actions. Duke Math. J. 62 (1991), 417-451. Zbl 0733.52006 MR 1104531

[7] J. Dugundji, Topology. Allyn and Bacon Inc., Boston 1966, Zbl 0144.21501 MR 0193606

[8] J. J. Duistermaat and J. A. C. Kolk, Lie groups. Universitext, Springer-Verlag, Berlin 2000. Zbl 0955.22001 MR 1738431

[9] M. Franz and V. Puppe, Exact cohomology sequences with integral coefficients for torus actions. Transform. Groups 12 (2007), 65-76. Zbl 05156780 MR 2308029

[10] M. Goresky, R. Kottwitz, and R. MacPherson, Equivariant cohomology, Koszul duality, and the localization theorem. Invent. Math. 131 (1998), 25-83. Zbl 0897.22009 MR 1489894

[11] V. Guillemin, Moment maps and combinatorial invariants of Hamiltonian $T^{n}$-spaces. Progr. Math. 122, Birkhäuser, Boston 1994. Zbl 0828.58001 MR 1301331

[12] A. Haefliger, Homotopy and integrability. In Manifolds-Amsterdam 1970 (Proc. Nuffic Summer School), Lecture Notes in Math. 197, Springer, Berlin 1971, 133-163. Zbl 0212.30704 MR 0278311 
[13] I. Hambleton and J.-C. Hausmann, Equivariant principal bundles over spheres and cohomogeneity one manifolds. Proc. London Math. Soc. (3) 86 (2003), 250-272. Zbl 1032.55015 MR 1971468

[14] Y. Karshon and S. Tolman, The moment map and line bundles over presymplectic toric manifolds. J. Differential Geom. 38 (1993), 465-484. Zbl 0791.58040 MR 1243782

[15] R. K. Lashof, Equivariant bundles. Illinois J. Math. 26 (1982), 257-271. Zbl 0458.55009 MR 650393

[16] R. Lashof, Equivariant bundles over a single orbit type. Illinois J. Math. 28 (1984), 34-42. Zbl 0554.55010 MR 730709

[17] R. K. Lashof and J. P. May, Generalized equivariant bundles. Bull. Soc. Math. Belg. Sér. A 38 (1986), 265-271 (1987). MR 885537

[18] R. K. Lashof, J. P. May, and G. B. Segal, Equivariant bundles with abelian structural group. In Proceedings of the Northwestern Homotopy Theory Conference (Evanston, Ill., 1982), Contemp. Math. 19, Amer. Math. Soc., Providence, RI, 1983, 167-176. Zbl 0526.55020 MR 0711050

[19] W. Lück, Transformation groups and algebraic K-theory. Lecture Notes in Math. 1408, Springer-Verlag, Berlin 1989. Zbl 0679.57022 MR 1027600

[20] W. Lück and B. Oliver, The completion theorem in $K$-theory for proper actions of a discrete group. Topology 40 (2001), 585-616. Zbl 0981.55002 MR 1838997

[21] A. T. Lundell and S. Weingram, The topology of CW-complexes. Van Nostrand Reinhold Co., New York 1969. Zbl 0207.21704

[22] P. S. Mostert, Local cross sections in locally compact groups. Proc. Amer. Math. Soc. 4 (1953), 645-649. Zbl 0103.01801 MR 0056614

[23] P. S. Mostert, Sections in principal fibre spaces. Duke Math. J. 23 (1956), 57-71. Zbl 0072.18102 MR 0075575

[24] N. Steenrod, The Topology of fibre bundles. Princeton Math. Ser. 14, Princeton University Press, Princeton, N. J., 1951. Zbl 0054.07103 MR 0039258

[25] T. E. Stewart, Lifting group actions in fibre bundles. Ann. of Math. (2) 74 (1961), 192-198. Zbl 0116.40502 MR 0126502

[26] T. tom Dieck, Faserbündel mit Gruppenoperation. Arch. Math. (Basel) 20 (1969), 136-143. Zbl 0188.28503 MR 0245027

[27] T. tom Dieck, Transformation groups. De Gruyter Stud. Math. 8, Walter de Gruyter \& Co., Berlin 1987. Zbl 0611.57002 MR 0889050

Received September 18, 2007; revised June 4, 2009

I. Hambleton, Department of Mathematics \& Statistics, McMaster University, Hamilton, ON L8S 4K1, Canada

E-mail: ian@math.mcmaster.ca

J.-C. Hausmann, Section de Mathématiques, Université de Genève, B.P. 240, CH-1211

Genève 24, Switzerland

E-mail: Jean-Claude.Hausmann@unige.ch 